\title{
Astrocytic extracellular vesicles modulate neuronal calcium homeostasis via transglutaminase-2
}

Elisa Tonoli ${ }^{1}$, Ivan Verduci ${ }^{2}$, llaria Prada ${ }^{3}$, Martina Gabrielli ${ }^{3}$, Greta Forcaia ${ }^{4}$, Clare Coveney ${ }^{5}$, Maria Pia Savoca ${ }^{1}$, David J. Boocock ${ }^{5}$, Giulio Sancini ${ }^{4,6}$, Michele Mazzanti ${ }^{2}$, Claudia Verderio ${ }^{3,6^{*}}$, Elisabetta A.M. Verderio ${ }^{1,7^{*}}$

${ }^{1}$ School of Science and Technology, Centre for Health, Ageing and Understanding of Disease, Nottingham Trent University, Nottingham NG11 8NS, United Kingdom.

${ }^{2}$ Department of Bioscience, University of Milan, Milano 20133, Italy.

${ }^{3}$ CNR Institute of Neuroscience, Vedano al Lambro 20854, Italy.

${ }^{4}$ Human Physiology Lab., School of Medicine and Surgery, University of Milano-Bicocca, Monza 20900, Italy.

${ }^{5}$ School of Science and Technology, The John van Geest Cancer Research Centre, Nottingham Trent University, Nottingham NG11 8NS, United Kingdom.

${ }^{6}$ NeuroMI (Milan Center for Neuroscience), School of Medicine and Surgery, University of Milano-Bicocca, Monza 20900, Italy.

${ }^{7} \mathrm{BiGeA}$, University of Bologna, Bologna 40126, Italy

*Elisabetta A.M. Verderio

School of Science and Technology, Nottingham Trent University

Clifton lane, Nottingham, NG11 8NS, United Kingdom

tel.: +44 (0)115 848 6628; fax: +44 (0)115848 6636

Email: elisabetta.verderio-edwards@ntu.ac.uk

\section{${ }^{*}$ Claudia Verderio}

CNR Institute of Neuroscience,

via Vanvitelli 32, 20129 Milan

tel.: +330250317011

Email: c.verderio@in.cnr.it 


\section{Abstract}

We have uncovered a novel role for astrocytes-derived extracellular vesicles (EVs) in controlling intraneuronal $\mathrm{Ca}^{2+}$ concentration $\left(\left[\mathrm{Ca}^{2+}\right]_{\mathrm{i}}\right)$ and identified transglutaminase-2 (TG2) as a surface-cargo of astrocytes-derived EVs. Incubation of hippocampal neurons with primed astrocyte-derived EVs have led to an increase in $\left[\mathrm{Ca}^{2+}\right]_{i}$, unlike EVs from TG2-knockout astrocytes. Exposure of neurons or brain slices to extracellular TG2 promoted a $\left[\mathrm{Ca}^{2+}\right]_{\mathrm{i}}$ rise, which was reversible upon TG2 removal and was dependent on $\mathrm{Ca}^{2+}$ influx through the plasma membrane. Patch-clamp and calcium imaging recordings revealed TG2-dependent neuronal membrane depolarisation and activation of inward currents, due to the opening of L-type-VOCCs and to $\mathrm{Na}^{+} / \mathrm{Ca}^{2+}$-exchanger (NCX) operation in the reverse mode, as indicated by VOCCS/NCX pharmacological inhibitors. A subunit of $\mathrm{Na}^{+} / \mathrm{K}^{+}$-ATPase was selected by comparative proteomics and identified as being functionally inhibited by extracellular TG2, implicating $\mathrm{Na}^{+} / \mathrm{K}^{+}$-ATPase inhibition in NCX reverse mode-switching leading to $\mathrm{Ca}^{2+}$ influx and higher basal $\left[\mathrm{Ca}^{2+}\right]_{\mathrm{i}}$. These data suggest that reactive astrocytes control intraneuronal $\left[\mathrm{Ca}^{2+}\right]_{i}$ through release of EVs with TG2 as responsible cargo, which could have a significant impact on synaptic activity in brain inflammation.

Keywords: astrocytes / calcium homeostasis / extracellular vesicles / hippocampal neurons / transglutaminase-2 


\section{Introduction}

The regulation of $\mathrm{Ca}^{2+}$ is a fundamental biological process in neurons, with $\mathrm{Ca}^{2+}$ signalling as the basis of synaptic plasticity, neurons survival and their ability to communicate (Catterall \& Few, 2008; Marambaud, DresesWerringloer et al., 2009). In neurons basal $\mathrm{Ca}^{2+}$ levels are tightly regulated within a narrow physiological range (Carafoli, 1987; Jones \& Keep, 1988), and a rise in intracellular $\mathrm{Ca}^{2+}$ concentration $\left(\left[\mathrm{Ca}^{2+}\right]_{i}\right)$ is the major trigger of neurotransmitter release from nerve endings. Propagation of action potentials and membrane depolarisation ( 20 $\mathrm{mV}$ ) produces $\mathrm{Ca}^{2+}$ influx through voltage-dependent $\mathrm{Ca}^{2+}$ channels which reaches micromolar levels at highly localised presynaptic sites in axons (Forti, Pouzat et al., 2000). Since the smallest change in $\mathrm{Ca}^{2+}$ currents can have a dramatic impact on neuronal function, $\mathrm{Ca}^{2+}$ influx is controlled by the action of $\mathrm{Ca}^{2+}$ membrane transporters at the plasma membrane, such as the $\mathrm{Ca}^{2+}$ pump ATPase and the sodium/calcium $\left(\mathrm{Na}^{+} / \mathrm{Ca}^{2+}\right)$ exchanger (NCX), and it is buffered by a large set of $\mathrm{Ca}^{2+}$ binding proteins which act as modulators of cellular $\mathrm{Ca}^{2+}$ transients. Extensive literature indicates that $\mathrm{Ca}^{2+}$ dyshomeostasis in neurons is linked with aging and neurodegeneration. However, mechanism(s) leading to $\left[\mathrm{Ca}^{2+}\right]_{i}$ alterations are still not completely clear (Nikoletopoulou \& Tavernarakis, 2012; Oh, Oliveira et al., 2013).

Transglutaminase-2 (TG2) is a $\mathrm{Ca}^{2+}$-dependent crosslinking enzyme known to be involved in multiple neurodegenerative diseases linked to $\mathrm{Ca}^{2+}$ dysregulation (Grosso \& Mouradian, 2012), as well as a variety of conditions triggered by inflammatory processes, such as wound-healing and tissue fibrosis (Verderio, Furini et al., 2015). The implication of TG2 was first suggested by the observation that the enzyme, in the presence of activating $\left[\mathrm{Ca}^{2+}\right]_{\text {i }}$, is able to crosslink pathogenic misfolded proteins typical of neurodegenerative conditions (e.g. amyloid- $\beta$ and $\alpha$-synuclein), thus favouring formation of protein aggregates (Andringa, Lam et al., 2004; Benilova, Karran et al., 2012; Grosso, Woo et al., 2014; Hartley, Zhao et al., 2008; Junn, Ronchetti et al., 2003). However a number of recent observations, such as the potentiation of $\mathrm{Ca}^{2+}$-induced hippocampal damage by TG2 in mice brain and higher sensitivity to kainic acid-induced seizures (Tucholski, Roth et al., 2006), as well as the neurotoxic role of astrocytic TG2 following acute brain injury (Feola, Barton et al., 2017; Monteagudo, Feola et al., 2018), have hinted to a possible role of TG2 in excitotoxicity-induced neuronal cell death, which may be either consequent to $\left[\mathrm{Ca}^{2+}\right]_{\mathrm{i}}$ increases or, as a new hypothesis, triggered by TG2-mediated changes in $\left[\mathrm{Ca}^{2+}\right]_{i}$. Astrocytes are an abundant source of TG2, which is externalised and accumulates in the extracellular matrix (ECM) in response to inflammatory stimuli (Pinzon, Breve et al., 2017). Importantly, astrocytes are key mediators 
of brain immune responses (Colombo \& Farina, 2016) and TG2 has been shown to play a role in neuroinflammation (lentile, Curro et al., 2015). Astrocytes control synapses either by direct contact and/or by secreted factors, released as single molecules or packaged into extracellular vesicles (EVs), which target preand post-synapses thus regulating synaptic behaviour (Farhy-Tselnicker \& Allen, 2018). In this study we have hypothesised that extracellular TG2 could disrupt $\mathrm{Ca}^{2+}$ homeostasis in neurons. We have uncovered a new role for extra-neuronal TG2, released by reactive astrocytes via EVs, in increasing neuronal $\left[\mathrm{Ca}^{2+}\right]_{\text {, }}$, and shown that this occurs through the opening of L-type VOCCs and NCX operation in the reverse mode, via regulation of $\mathrm{Na}^{+} / \mathrm{K}^{+}$-ATPase activity. This process is a novel example of neuron-regulation by astrocytes-derived EVs cargo under both physiological and neuroinflammatory conditions. 


\section{Results}

\section{TG2 released by primary astrocytes in association with EVs increases $\left[\mathrm{Ca}^{2+}\right]_{i}$ in neurons}

Previous studies have shown that astrocytes are a main source of extracellular TG2 in the brain, particularly in situations of inflammation/astroglial response to injury (Colak \& Johnson, 2012; Monteagudo et al., 2018; Pinzon et al., 2017; Quinn, Yunes-Medina et al., 2018). A granular pattern of TG2 distribution was detected by immunofluorescence in either permeabilized or non-permeabilized fixed cultures of astrocytes, suggesting that TG2 has a predominant extracellular location in astrocytes (Fig. 1A). Evaluation of endogenous cell surface TG2 by an optimised activity assay revealed high extracellular TG2 activity (sensitive to the specific inhibitor ZDON) in cultured astrocytes (Fig. EV1). Conversely in hippocampal neurons, TG2 showed a significantly higher staining in permeabilized cells (Fig.1B), where it partially localised at synaptic sites, as indicated by co-staining with the preand post-synaptic markers VGLUT1 (blue) and SHANK2 (red) (Fig. 1C). Western blotting analysis of crude synaptosomes from adult mouse brain, revealed a significant enrichment of TG2 at synaptic contacts (Fig. 1D), confirming the presence of TG2 at synaptic sites in vivo, either in neuron terminals (validated by the expression of NR2B, PSD95, VGAT and VGLUT1) or in the astrocytic processes wrapping the synapses (validated by GFAP expression) (Fig. 1A and 1D) (Gylys, Fein et al., 2000).

Once externalised at the astrocyte surface TG2 can bind to matrix fibronectin (FN) (Pinzon et al., 2017) and our data confirmed a partial co-localisation of TG2 with FN (Fig. EV2). We hypothesised that TG2 may be released via extracellular vesicles (EVs), as recently described in other cell systems (Antonyak, Li et al., 2011; DiazHidalgo, Altuntas et al., 2016; Furini, Schroeder et al., 2018). In this form, TG2 could travel to neurons, unless released from astrocytes in direct contact with synapses. To test this possibility, astrocyte-derived EVs were isolated from conditioned medium of primary astrocytes obtained from wild type $\left(\mathrm{TGM}^{+/+}\right)$and knockout $\left(\mathrm{TGM}^{-/-}\right)$ mouse brain (De Laurenzi \& Melino, 2001). Characterisation by Nanoparticle tracking analysis (Fig. 2A), TEM (Fig. 2B), detection of vesicular markers ALIX and Flotillin-2 and absence of negative marker TOM20 confirmed the size, morphology and vesicular cargo of EVs (Fig. 2C). NTA showed a trend increase in EVs number upon LPS treatment (Fig. 2A). Neither average particle number nor mode size were significantly different between WT and TG2KO cells, either untreated or LPS-treated (Fig. 2A-I and 2A-II), whereas a significant difference was observed between WT and WT+LPS particle number when looking at specific sizes (45-195 nm range) (Fig. 2AIII). TEM analysis and immunogold labelling of WT vesicles showed that they had the expected cup shape and 
confirmed the presence on the EVs surface of specific EVs marker CD63 and of TG2 (Fig. 2B). Western blotting analysis of astrocytes-derived EVs lysates revealed the presence of TG2 protein in WT EVs which displayed a trend increase upon pro-inflammatory stimulation with LPS (Fig. 2C). Furthermore, negligible TG2 was detected in vesicle-free supernatants after recovering all proteins by TCA precipitation, suggesting a prominent vesicular localisation of TG2 in primary astrocytes (Fig. 1A). These data show for the first time that TG2 is released from cultured astrocytes as a cargo of EVs under both resting physiological and stimulated (inflammatory) conditions. The localisation of TG2 in EVs was investigated using a sensitive enzymatic assay as we previously described (Furini et al., 2018). TG2 was detected at comparable levels in both intact and lysed EVs suggesting a predominant EVs surface location (Fig. 2D). To investigate the involvement of the astrocytes EVs TG2 cargo on neuronal function, the modulation of $\left[\mathrm{Ca}^{2+}\right]_{i}$ by EVs isolated from WT and TG2KO LPS-primed astrocytes was monitored in fura-2-loaded hippocampal neurons. In order to exclude variations in $\left[\mathrm{Ca}^{2+}\right]_{i}$ due to vesicular ATP leaking from astrocyte-derived EVs (D'Arrigo, Gabrielli et al., 2021), the assay was performed in the presence of the ATP degrading enzyme apyrase. As shown in Fig. 2E, TG2-containing EVs (EVs WT) were able to rise basal $\left[\mathrm{Ca}^{2+}\right]_{\mathrm{i}}$, whereas addition of TG2KO EVs did not cause significant changes. These data have two novel implications: that astrocytes-derived EVs concur to the regulation of neuronal $\mathrm{Ca}^{2+}$ homeostasis and that TG2, which is exposed on the surface of astrocytes-derived EVs, is responsible for this specific function.

\section{Extracellular TG2 increases cytoplasmic calcium concentration, both in cultured neurons and hippocampal slices}

To gain insights into the mechanism leading to increased neuronal $\left[\mathrm{Ca}^{2+}\right]_{\mathrm{i}}$ by extracellular TG2 we next monitored neuronal $\left[\mathrm{Ca}^{2+}\right]_{i}$ dynamics upon addition to the extracellular solution of purified TG2. Differentiated hippocampal neurons often exhibit synchronous $\mathrm{Ca}^{2+}$ oscillations in the soma, reflecting burst of neuronal firing (Bacci, Verderio et al., 1999). Incubation with purified TG2 promoted the onset of a synchronous $\mathrm{Ca}^{2+}$ spike and increased the interspike $\left[\mathrm{Ca}^{2+}\right]_{\text {, }}$ leading to blockage of oscillatory activity (Fig. 3A-I, "TG2"). Upon TG2 removal by wash with $\mathrm{KRH}$ (Fig. 3A-I, "Wash"), interspike $\left[\mathrm{Ca}^{2+}\right]_{i}$ decreased to pre-treatment values and spontaneous $\mathrm{Ca}^{2+}$ oscillations re-started, indicating that the action of soluble TG2 was reversible. To further examine the action of TG2 on $\left[\mathrm{Ca}^{2+}\right]$, neurons were exposed to TG2 in the presence of TTX $(1 \mu \mathrm{M})$, which prevents spontaneous synaptic and synchronous $\mathrm{Ca}^{2+}$ activity. In virtually all neurons tested, addition of TG2 led to a small but highly significant 
increase in $\left[\mathrm{Ca}^{2+}\right]_{i}$ in the neuron cell bodies (Fig. 3A-II, "TG2"), while no $\left[\mathrm{Ca}^{2+}\right]_{i}$ changes were observed in astrocytes present in the cultures. To rule out that the phenomenon was only found in dissociated cultures, we investigated the effect of TG2 on hippocampal slices. Similar $\mathrm{Ca}^{2+}$ response was evoked by TG2 in neurons in mouse hippocampal slices loaded with fura-2 when the TG2 protein was added in perfusion mode (Fig. 3B). In order to clarify whether extracellular TG2 increases basal $\mathrm{Ca}^{2+}$ levels by enhancing $\mathrm{Ca}^{2+}$ influx into neurons, we exposed cultured neurons to purified TG2 in $\mathrm{Ca}^{2+}$-free solution (Fig. $3 \mathrm{C}-\mathrm{I}$ ). No rise in basal $\left[\mathrm{Ca}^{2+}\right]_{i}$ occurred in $\mathrm{Ca}^{2+}$-free conditions, while TG2-dependent $\mathrm{Ca}^{2+}$ rises were clearly recorded from the same neurons after addition of $\mathrm{Ca}^{2+}$ ions $\left(2 \mathrm{mM} \mathrm{Ca}^{2+}\right)$ to the extracellular medium (Fig. 3C-II). This establishes that the $\left[\mathrm{Ca}^{2+}\right]_{i}$ rise evoked by exogenous TG2 is mediated by $\mathrm{Ca}^{2+}$ influx from the extracellular environment through the plasma membrane. Interestingly, the action of TG2 was reversible, as observed in the absence of TTX (Fig. 3A-I) and neurons recovered basal $\left[\mathrm{Ca}^{2+}\right]_{i}$ upon removal of the protein ("Wash") from the extracellular solution (Fig. 3A-I, 3A-II and 3B). This finding implies that the observed process might not be due to transamidation, which leads to a covalent irreversible modification of protein substrates and should not disappear once TG2 is removed. To analyse this further, we tested the activity of exogenous TG2 added to hippocampal neurons during calcium imaging recordings and found that it was catalytically inactive in the same experimental conditions of the calcium imaging, unless activated by supplementation of a reducing agent (Fig. EV3). Notably, we previously showed that extracellular TG2 bound to the ECM is catalytically inactive or poorly active in the absence of a reducing agent (Verderio, Telci et al., 2003).

We next asked whether TG2 secreted/externalised from neurons could rise basal $\left[\mathrm{Ca}^{2+}\right]_{\mathrm{i}}$ similarly to exogenous/astrocyte-derived TG2. To this aim, we first transfected hippocampal neurons with TG2 and measured $\left[\mathrm{Ca}^{2+}\right]_{\mathrm{i} .}$ Transfected cells (pEGFP-N1-TG2) displayed an increase in resting $\left[\mathrm{Ca}^{2+}\right]_{\mathrm{i}}$ compared to control neurons (pEGFP-N1) (Fig. 4A). This suggests that upon over-expression neuronal TG2 may act extracellularly in an autocrine manner, controlling basal $\left[\mathrm{Ca}^{2+}\right]_{\text {, }}$ albeit we cannot exclude an intracellular action of the protein. Treatment of the transfected cells with BOCDON, a non-permeable inhibitor of TG2 targeting extracellular TG2, did not affect changes in $\left[\mathrm{Ca}^{2+}\right]_{i}$ evoked by the transfected TG2 (Fig. 4B). As testing the transamidating activity of TG2 solubilised in the extracellular saline confirmed that extracellular TG2 is predominantly inactive unless treated with a reducing agent (Fig. EV3), and that inhibition of extracellular TG2 does not affect intracellular $\mathrm{Ca}^{2+}$ changes, we conclude that TG2 evoking $\mathrm{Ca}^{2+}$ responses is prevalently inactive as a protein crosslinker. 


\section{Extracellular TG2 causes calcium influx through L-type VOCCs}

We next asked how extracellular TG2 promotes $\mathrm{Ca}^{2+}$ influx through the plasma membrane. Neurons were stimulated with exogenous TG2 in the presence of blockers of the main $\mathrm{Ca}^{2+}$ entry pathways in neurons, namely glutamate receptors and VOCCs. The NMDA receptor antagonist APV and the AMPA/kainate receptor antagonist CNQX did not affect $\mathrm{Ca}^{2+}$ increase upon TG2 addition, ruling out the involvement of these ligand-gated $\mathrm{Ca}^{2+}$ permeable receptors in the process (Fig. 5A-I and 5A-II). To analyse the possible contribution of VOCCs, we used a selection of pharmacological inhibitors of these channels. Pre-treatment with cadmium, a general blocker of VOCCs, reduced TG2-dependent $\mathrm{Ca}^{2+}$ responses by about $82.4 \%$ (Fig. 5B-I) and caused an almost complete recovery $(88.3 \%)$ of $\left[\mathrm{Ca}^{2+}\right]_{i}$ towards basal levels when applied during the plateau phase of $\mathrm{Ca}^{2+}$ response induced by exogenous TG2 (Fig. 5C-I). Similarly, pre-treatment with nickel, a more specific inhibitor of T-type VOCCs, decreased TG2-dependent $\mathrm{Ca}^{2+}$ rises by $66.2 \%$ (Fig. 5B-II) while it caused a drop of $\left[\mathrm{Ca}^{2+}\right]_{i}$ below basal level (151\% inhibition) when applied during the plateau phase (Fig. 5C-II). The involvement of VOCCs was further indicated by the observation that $\mathrm{Ca}^{2+}$ transients evoked by depolarisation $(15 \mathrm{mM} \mathrm{KCl}$ ) rose faster and reached a higher peak in the presence of extracellular TG2, with a significant increase in average $\mathrm{Ca}^{2+}$ influx (Fig. 5D). Among VOCCs controlling $\mathrm{Ca}^{2+}$ transport through the plasma membrane, L-type VOCCs are highly abundant in the somatodendritic region of hippocampal neurons (Condliffe, Corradini et al., 2010; Leitch, Szostek et al., 2009; Pravettoni, Bacci et al., 2000). We therefore asked whether these channels might contribute to $\mathrm{Ca}^{2+}$ influx in the neuronal soma evoked by exogenous TG2. We found that $\mathrm{Ca}^{2+}$ responses to TG2 were reduced by about $36 \%$ in neurons pre-treated with the selective of L-type blocker nifedipine (NF) (Fig. 5E-I). In addition, the drug caused a partial recovery of $\left[\mathrm{Ca}^{2+}\right]_{i}$ towards resting levels when applied during the plateau phase of $\mathrm{Ca}^{2+}$ response induced by TG2 (Fig. 5E-II), suggesting that TG2 dependent $\mathrm{Ca}^{2+}$ influx partially occurs through L-type VOCCs.

\section{Extracellular TG2 induces membrane depolarisation and the generation of ionic inward currents, which}

\section{are inhibited by Nifedipine}

To further characterise the $\left[\mathrm{Ca}^{2+}\right]_{\mathrm{i}}$ response evoked by TG2, we performed whole cell electrophysiological recordings of hippocampal neurons. In current clamp, TG2 perfusion induced a slow membrane depolarisation (of about $20 \mathrm{mV}$ ) (Fig. 6A-I), which was reverted by addition of nickel (Fig. 6A-II). In voltage clamp, TG2 promoted 
excitatory postsynaptic currents (EPSCs), consistent with the activation of an inward $\mathrm{Ca}^{2+}$ current (Fig. 6B). In the same configuration, analysis of the current/voltage relationship (I/V curves) using a protocol for isolation of L-type VOCCs, showed that perfusion of TG2 (Fig. 6C-I, red I/V curve) led to an increased inward current compared to control (black I/V curve), which was completely prevented by the presence of the L-type VOCCs blocker NF (Fig. 6C-II, red I/V curve compared to control black I/V curve). These data revealed L-type VOCCs as one of the possible main targets of TG2 responsible for dysregulation of basal $\mathrm{Ca}^{2+}$ concentration in neurons.

\section{Contribution of the sodium/calcium exchanger to the calcium response evoked by TG2}

Data so far have identified L-type VOCCs as the main channel through which extracellular TG2 induces $\mathrm{Ca}^{2+}$ influx and membrane depolarisation. Despite electrophysiological recordings indicated complete inhibition of inward $\mathrm{Ca}^{2+}$ current evoked by TG2 under block of L-type VOCCs (Fig. 6C), a residual $\mathrm{Ca}^{2+}$ response was observed in $\mathrm{Ca}^{2+}$ imaging experiments, largely sensitive to both cadmium and nickel (Fig. $5 \mathrm{E}$ ). In addition to VOCCs, both cadmium and nickel are known to inhibit the activity of the $\mathrm{Na}^{+} / \mathrm{Ca}^{2+}$-exchanger (NCX), a key regulator of $\mathrm{Ca}^{2+}$ transport through the plasma membrane. $\mathrm{NCX}$ normally removes $\mathrm{Ca}^{2+}$ from neurons in exchange for $\mathrm{Na}^{+}$, which enters the neuron down its gradient across the plasma membrane (Blaustein \& Lederer, 1999). However, perturbation of the $\mathrm{Na}^{+}$gradient leads to operation of $\mathrm{NCX}$ in the reverse mode, causing $\mathrm{Ca}^{2+}$ influx into the neurons (Blaustein \& Lederer, 1999). To investigate the involvement of NCX in the reverse mode in TG2-induced $\mathrm{Ca}^{2+}$ influx, we removed $\mathrm{Na}^{+}$(and potassium $\mathrm{K}^{+}$) from the extracellular saline. In these conditions, the response to extracellular TG2 was strikingly increased (about 8-fold increase) (Fig. 7A), suggesting that NCX may amplify TG2-dependent $\mathrm{Ca}^{2+}$ entry. Conversely, addition of the NCX inhibitor YM-244769 significantly decreased TG2-dependent $\mathrm{Ca}^{2+}$ influx in both normal and $\mathrm{Na}^{+}$-free medium (Fig. 7B), further supporting NCX contribution to $\mathrm{Ca}^{2+}$ elevations in neurons exposed to TG2.

\section{Interactors of extracellular TG2 in hippocampal neurons: $\mathrm{Na}^{+} / \mathrm{K}^{+}-\mathrm{ATPase}$ inhibition by extracellular TG2.}

To gain insights into the molecular interactions leading to the opening of L-type VOCCs and NCX operation by extracellular TG2 in neurons, we immunoprecipitated TG2 from the lysate and secretome of neurons treated with extracellular TG2 and analysed the immunoprecipitated proteins by quantitative comparative proteomics relative to untreated neurons immunoprecipitates as shown in Fig. 8A (full list of proteins is available in Table EV1). 
Among the interactors of extracellular TG2 (Fig. 8B) the finding of vesicle-associated membrane protein 2 (VAMP2) and alpha-soluble NSF attachment protein (SNAA) (confidence $>70 \%$ ), suggests that extracellular TG2 localises at synapses similarly to the endogenous protein (Fig. 1). The association of $\mathrm{Ca}^{2+}$ buffer proteins calretinin, calbindin and a subunit of $\mathrm{Ca}^{2+} /$ calmodulin-dependent kinase with extracellular TG2 is consistent with the ability of extracellular TG2 to elicit $\left[\mathrm{Ca}^{2+}\right]_{i}$ changes in neurons. Among the transporters which emerged as significant TG2 partners, $\mathrm{Na}^{+} / \mathrm{K}^{+}$-transporting ATPase subunit a (AT1A3) was three times more associated with neurons exposed to extracellular TG2 (confidence 70\%) (Fig. 8B). We hypothesised that AT1A3 could be part of the mechanism of $\mathrm{Ca}^{2+}$ influx triggered by extracellular TG2 via inhibition of $\mathrm{Na}^{+} / \mathrm{K}^{+}$-ATPase by TG2, with increase of intracellular $\mathrm{Na}^{+}$leading to switch of $\mathrm{NCX}$ in the reverse mode (Fig 7A-B). To test ATPase inhibition by TG2, ATPase activity was measured using ATPase isolated from porcine cerebral cortex either in the presence or absence of TG2 using the specific $\mathrm{Na}^{+} / \mathrm{K}^{+}$-ATPase inhibitor ouabain as a control. Incubation with TG2 led to a small but significant reduction in ATPase activity suggesting that perturbation of $\mathrm{Na}^{+} / \mathrm{K}^{+}$-transport by extracellular TG2 may trigger the rise in neuronal $\mathrm{Ca}^{2+}$ influx (Fig $8 \mathrm{C}$ ).

According to our findings, reactive astrocytes control intraneuronal $\left[\mathrm{Ca}^{2+}\right]_{\mathrm{i}}$ through release of TG2 in association to extracellular vesicles (Fig. 9). Upon interaction with neurons extracellular TG2 promotes the opening of L-type calcium channels and induces the $\mathrm{Na}^{+} / \mathrm{Ca}^{2+}$-exchanger to operate in the reverse mode, likely through inhibition of $\mathrm{Na}^{+} / \mathrm{K}^{+}$-ATPase, setting basal $\left[\mathrm{Ca}^{2+}\right]_{\mathrm{i}}$ at higher levels and enhancing neurotransmission. These findings link astrogliosis, which typically occurs under brain inflammation and degeneration with $\mathrm{Ca}^{2+}$ dyshomeostasis in neurons and identifies extracellular TG2 as a key astrocyte-derived factor driving neuron dysfunction. 


\section{Discussion}

Our study unveils a novel mechanism by which TG2 regulates $\mathrm{Ca}^{2+}$ homeostasis in hippocampal neurons. We show that extracellular TG2 drives $\mathrm{Ca}^{2+}$ influx into neurons both in culture and brain slice, via inhibition of $\mathrm{Na}^{+} / \mathrm{K}^{+}-$ ATPase activity and consequent activation of L-type VOCCS and NCX operating in the reverse mode.

A key observation is that the sources of extracellular TG2 are astrocytes-derived EVs, which have TG2 as cargo displayed at the EVs surface. EVs-associated TG2 significantly increases $\left[\mathrm{Ca}^{2+}\right]_{\mathrm{i}}$ in neurons, as demonstrated by selective $\left[\mathrm{Ca}^{2+}\right]_{i}$ rises in response to EVs derived from WT but not TG2KO cells. Notably, TG2 content tends to increase in EVs released by reactive astrocytes. This is consistent with published data showing higher externalisation of astrocytic TG2 in response to neuroinflammation (i.e. TNF- $\alpha+$ IL-1 $\beta$ treatment) (Pinzon et al., 2017) and increased TG2 levels in association with astrogliosis, an inflammatory processes which is activated in brain in response to injury and the release of pro-inflammatory cytokines (Hostenbach, Cambron et al., 2014; lentile et al., 2015). Astrocytes play a key role in synaptic scaling, i.e. the adjustment of synaptic strength in response to prolonged changes in the electrical activity to maintain neuronal function and survival (Papouin, Dunphy et al., 2017). Potentiation of synaptic strength largely occurs via insertion of postsynaptic AMPA receptors driven by the release of TNFa by glial cells (Stellwagen \& Malenka, 2006). However, the exact mechanism and molecular actors involved in synaptic scaling are still unknown (Papouin et al., 2017). By enhancing neuronal $\left[\mathrm{Ca}^{2+}\right]$, TG2 released in association with astrocytic EVs may favour excitatory transmission, thereby contributing to synaptic scale up. Further work is required to address this intriguing hypothesis.

EVs play an emerging role in neuron-glia cross-talk (Holm, Kaiser et al., 2018; Turola, Furlan et al., 2012). In particular, astrocyte-derived EVs induce a long lasting potentiation of spontaneous excitatory transmission (Antonucci, Turola et al., 2012; Gabrielli, Battista et al., 2015; You, Borgmann et al., 2020) but upon chronic exposure EVs from reactive astrocytes exert detrimental effects on synapse stability (Prada, Gabrielli et al., 2018), neurite differentiation and neuronal firing (You et al., 2020). Astrocyte-derived EVs isolated from plasma of Alzheimer's disease $(A D)$ patients carry high levels of complement effector proteins compared to healthy controls, suggesting that they could promote the spreading of inflammatory signals (Goetzl, Schwartz et al., 2018). By showing that TG2 sorted on the surface of astrocytic EVs has the capacity to regulate neuronal $\mathrm{Ca}^{2+}$ homeostasis, we add an important piece of information to decipher the complex astrocyte-to-neuron signalling mediated by EVs under physiology and neuroinflammation. 
The action of TG2 displayed in astrocytes-derived EVs is also reproduced by soluble extracellular TG2. TG2dependent $\mathrm{Ca}^{2+}$ influx sets basal $\left[\mathrm{Ca}^{2+}\right]_{i}$ at higher levels and promotes spontaneous EPSCs. This is a novel function that has never been attributed to TG2 before. Interestingly, $\left[\mathrm{Ca}^{2+}\right]_{\mathrm{i}}$ is restored to basal levels upon removal of TG2 from the extra-neuronal solution, revealing that the process is reversible and not linked to neuron damage. Reversibility of TG2 action suggests also that conformation rather than transamidating activity of the enzyme regulates $\left[\mathrm{Ca}^{2+}\right]_{\mathrm{i}}$ homeostasis, as covalent crosslinking of a substrate would lead to irreversible effects. Several past and present evidences suggest that TG2 is capable of crosslinking-independent modifications (Akimov, Krylov et al., 2000; Stephens, Grenard et al., 2004; Steppan, Bergman et al., 2017; Verderio et al., 2003) and TG2 conformation is important in mediating the cellular response (Katt, Antonyak et al., 2018). In mouse striatal cells, TG2 in open conformation was shown to have cytotoxic effects, increasing the cells susceptibility to oxygen/glucose deprivation-mediated cell death. However, the underlying mechanism remains largely unknown (Colak, Keillor et al., 2011; Singh, Zhang et al., 2016).

Consistent with a crosslinking-independent role of TG2 in the control of $\mathrm{Ca}^{2+}$ dynamics, in vitro measurement of purified TG2 activity in $\mathrm{KRH}$, mimicking the extracellular solution used for $\mathrm{Ca}^{2+}$ and electrophysiological recordings, showed that the protein is mostly inactive under this experimental condition. Moreover, a specific extracellular TG2 inhibitor (BOCDON) does not affect extracellular TG2-mediated $\left[\mathrm{Ca}^{2+}\right]_{i}$ changes. On the other hand, we cannot exclude that the enzyme transamidating activity may contribute, in part, to the control of neuronal $\mathrm{Ca}^{2+}$ homeostasis. For example, it could be hypothesised that residual TG2 activity may act on substrates present in the extracellular medium that could promote $\mathrm{Ca}^{2+}$ influx upon transamidation and which would be removed together with TG2 during the washing step, thus resulting in reversibility. In this respect, transamidation of monoamine neurotransmitters, a reaction called monoaminylation, is a less characterised function of TG2 that may play a role in the process (Muma, 2018). Therefore, further work is required to exclude the possible contribution of TG2 activity to the control of $\mathrm{Ca}^{2+}$ dynamics.

Importantly, we show that in physiological solution TG2 induces a small increase in neuronal $\left[\mathrm{Ca}^{2+}\right]_{\text {, }}$ not associated to cell death, setting $\left[\mathrm{Ca}^{2+}\right]_{i}$ to levels typically displayed by astrocytes in primary culture, that are significantly higher compared to neurons. Such increase in $\left[\mathrm{Ca}^{2+}\right]$, is functionally relevant as it promotes neuronal transmission (spontaneous EPSCs). This suggests that TG2 may exert a fundamental homeostatic function in 
neurons, promoting neurotransmission, not only contributing to neurodegenerative and neuroinflammatory processes upon $\mathrm{Ca}^{2+}$ overload (Grosso \& Mouradian, 2012).

Identification of L-type VOCCs and NCX as the TG2 molecular targets mediating $\mathrm{Ca}^{2+}$ influx and setting basal $\left[\mathrm{Ca}^{2+}\right]_{i}$ at a higher levels in neurons, provides mechanistic understanding of the pathway. Both molecular targets have been identified using pharmacological blockers combined to $\mathrm{Ca}^{2+}$ and electrophysiological recordings. Interestingly, activation of L-type VOCCs is important for synaptic plasticity (Grover \& Teyler, 1990; Raymond \& Redman, 2002) such as long-term potentiation (LTP), suggesting that through this target TG2 may have a role in physiological brain functions. However, alterations in VOCCs activity have been also reported in neurodegeneration, such as in AD (LaFerla, 2002; Nimmrich \& Eckert, 2013), suggesting that persistent activation of VOCCs may also contribute to degenerative processes. Similarly to VOCCs, NCX is fundamental for the regulation of $\left[\mathrm{Ca}^{2+}\right]_{i}$ and activation of the reverse mode $\left(\mathrm{Ca}^{2+}\right.$ influx $)$ has been associated with neuropathic pain caused by nerve-injury (Jaggi \& Singh, 2011), ischemic damage (Matsuda, Arakawa et al., 2001), increased oxygen/glucose deprivation-mediated neuronal damage and infarct injury (Secondo, Pignataro et al., 2015). Thus, sustained elevations of TG2 in the extracellular environment, via opening of L-type VOCCs and NCX reverse operation, could participate to general dysregulation of $\mathrm{Ca}^{2+}$ homeostasis in neurodegenerative conditions. Analysis of the TG2 interactome has identified a subunit of $\mathrm{Na}^{+} / \mathrm{K}^{+}$-transporting ATPase as partner of extracellular TG2, and our data suggest that its inhibition by TG2 could reverse the activity of NCX, thus leading to $\mathrm{Ca}^{2+}$ inward flux and the opening of L-type VOCCs.

In conclusion, our data highlight for the first time a specific function of extracellular TG2, either released by astrocytes via EVs or in soluble form, on $\mathrm{Ca}^{2+}$ homeostasis in neurons, through opening of L-type VOCCs and NCX operation in the reverse mode, likely mediated by the $\mathrm{Na}^{+} / \mathrm{K}^{+}$-transporting ATPase (Fig. 8). This is the first evidence that astrocytes control intraneuronal $\mathrm{Ca}^{2+}$ through release of TG2 in association with EVs. 


\section{Materials and Methods}

Primary cultures: primary cultures of hippocampal neurons were established from E18 Sprague Dawley rat embryos as previously described (Gabrielli et al., 2015). After 14-21 days in vitro (DIV), the cultures contain a significant percentage of astrocytes (Verderio, Bacci et al., 1999). Primary astrocytes from C57BL/6 WT and TG2 knockout (TG2 ${ }^{-/}$or TG2KO) P3 mouse pups were established as previously described (Gabrielli et al., 2015). TG2KO mice were originally obtained from Gerry Melino (De Laurenzi \& Melino, 2001) and fully backcrossed as previously described (Scarpellini, Huang et al., 2014). For TG2 cell-surface activity assay, primary astrocytes were obtained from BrainBits (BrainBits, Springfield, IL, USA) and processed following the manufacturer's protocol.

Reagents: guinea pig liver TG2 (Zedira GmbH, Darmstadt, Germany) 10-30 $\mu \mathrm{g} / \mathrm{ml}$; LPS (Sigma-Aldrich, St. Louis, MO, USA) $1 \mu \mathrm{g} / \mathrm{ml}$; TTX (Tocris, Bristol, United Kingdom) $1 \mu \mathrm{M}$; BOCDON (Zedira) $200 \mu \mathrm{M}$; Nifedipine (SigmaAldrich) $1 \mu \mathrm{M}$; Cadmium (Sigma-Aldrich) $200 \mu \mathrm{M}$; Nickel (Sigma-Aldrich) $100 \mu \mathrm{M}$; YM-244769 (Tocris) $1 \mu \mathrm{M}$; APV (Tocris) $50 \mu \mathrm{M}$; CNQX (Tocris) $10 \mu \mathrm{M}$; biotin-cadaverine (Sigma-Aldrich) $0.1 \mathrm{mM}$; apyrase (Sigma-Aldrich) 30 $\mathrm{U} / \mathrm{ml}$; human fibronectin (Sigma-Aldrich) $5 \mu \mathrm{g} / \mathrm{ml}$; ATPase from porcine cerebral cortex (Sigma-Aldrich) $0.5 \mathrm{mU}$; Ouabain (Tocris) $1 \mathrm{mM}$.

Immunocytochemical staining: Cells were fixed in $4 \%$ paraformaldehyde - $4 \%$ sucrose (w/v) and immunofluorescence staining was performed using the following antibodies: mouse monoclonal anti-TG2 (IA12 Tim Johnson, University of Sheffield (Scarpellini et al., 2014)), guinea pig anti-VGLUT1 (Synaptic System, Goettingen, Germany), rabbit anti-GFAP (Dako, Agilent, Santa Clara, CA, USA), rabbit anti-Shank2 (Synaptic System), rabbit anti-Fibronectin (Sigma-Aldrich), rabbit anti- $\beta$-tubulin (Sigma-Aldrich) and rabbit anti-NR2B (Alomone, Jerusalem, Israel). Secondary antibodies were conjugated with Alexa-488, Alexa-555 and Alexa-633 fluorophores (Invitrogen). Further details are available in the Appendix Table S1. Coverslips were visualised by laser scanning Leica SP5 confocal microscope using 63X oil immersion objective. Successive serial optical sections $(0.5 \mu \mathrm{m})$ were recorded over $5 \mu \mathrm{m}$ planes and maximum projections were selected for quantification. Fluorescence intensity and co-localisation were estimated using the Leica LAS AF Lite software or ImageJ software as indicated in the Appendix Extended methods.

Crude synaptosomes preparation: Two adult mouse cortices were homogenised in $10 \mathrm{ml}$ of cold SHE buffer (4 mM HEPES pH 7.3, 320 mM sucrose, 1 mM EGTA, protease inhibitors), using a glass-Teflon homogeniser on 
ice. Brain homogenates were then centrifuged $1000 \mathrm{xg}$ for $10 \mathrm{~min}$ at $4^{\circ} \mathrm{C}$ to obtain the Low speed supernatant (LSS). A part of LSS was saved for analysis, while the remaining volume was centrifuged at $12500 x g$ for 20 min at $4^{\circ} \mathrm{C}$ to obtain the crude synaptosomes (pellet). Pelleted synaptosomes were washed once in SHE (10 ml/brain) and centrifuged again at $12500 \times \mathrm{xg}$ for $20 \mathrm{~min}$ at $4^{\circ} \mathrm{C}$. The final pellet (crude synaptosomal fraction) was resuspended in $1 \mathrm{ml} \mathrm{SHE} \mathrm{buffer} \mathrm{and} \mathrm{quantified} \mathrm{by} \mathrm{BCA} \mathrm{(Sigma-Aldrich).}$

Western blotting: Samples were electrophoresed on 10\% SDS polyacrylamide gels according to standard procedures. The following antibodies were used: mouse monoclonal anti-TG2 (IA12), anti-FLOT-2 (BD Biosciences, Wokingham, United Kingdom), anti-PSD-95 (Neuromab, Davis, C, USA), anti-TOM20 (Santa Cruz,

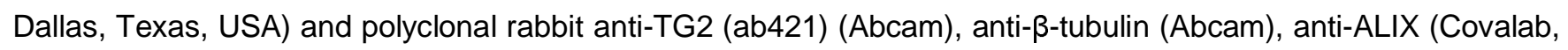
Bron, France), anti-NR2B (Alomone, Jerusalem, Israel), anti-VGAT (Synaptic systems, Goettingen, Germany), anti-VGLUT1 (Synaptic systems), anti-GFAP (GeneTex). Further details are available in Appendix Table S1. Blots were developed using enhanced chemiluminescence (SuperSignal West Femto Maximum Sensitivity Substrate, Thermo Fisher Scientific) and protein band intensity was quantified by Aida Image Analyser v.4.03 (Raytest, Germany).

Isolation of extracellular vesicles (EVs): EVs were isolated from conditioned medium (CM) of astrocytes by differential centrifugation as previously described (Furini et al., 2018; Prada et al., 2018). The 10 000xg centrifugation was omitted to pellet large and small EVs together. Each astrocytes preparation derived from $\geq 10$ mouse P3 pups. Specifically, 80\% confluent monolayers were washed twice with PBS or KRH and incubated in serum-free media in the presence or absence of lipopolysaccharide (LPS, $1 \mu \mathrm{g} / \mathrm{ml}$ ), for $24 \mathrm{~h}$. CM was collected and supplemented with protease inhibitors before centrifugation. EVs were resuspended either intact in particlefree PBS (for Nanoparticle Tracking Analysis, TEM and TG2 activity assay) or KRH (for $\mathrm{Ca}^{2+}$ imaging) or lysed in RIPA buffer (for western blotting) or mild lysis buffer (0.25 M sucrose, $2 \mathrm{mM}$ EDTA, $5 \mathrm{mM}$ Tris- $\mathrm{HCl}$, pH 7.4) (for TG2 activity assay).

Nanoparticle tracking analysis: Nanoparticle tracking analysis (NTA) was performed using ZetaView PMX 120 (Particle Metrix, Meerbusch, Germany) and its corresponding software (ZetaView 8.04.02) to examine EVs size distribution and concentration. EVs samples, resuspended in particle-free PBS, were diluted to reach 50-200 particles/frame and analysed with a flow cell sensitivity of $80 \%$ across two cycles of 11 positions/cycle. 
Transmission Electron Microscopy: EVs isolated from untreated WT astrocytes were analysed by Transmission Electron Microscopy (TEM) as previously described (Charrin, Palmulli et al., 2020), with a few modifications. Briefly, EVs were deposited for $20 \mathrm{~min}$ on formvar/coated 200 mesh nickel EM grids (TAAB Laboratories Equipment Ltd, Berks, United Kingdom). Then grids were fixed with $2 \%$ paraformaldehyde/phosphate buffer $\mathrm{pH} 7.4$ and processed for negative staining or single immunogold labelling. EVs were stained with mouse monoclonal anti-CD63 (Abcam) or anti-TG2 antibody (IA12) followed by $6 \mathrm{~nm}$ colloidal gold anti-mouse IgG (Jackson Immunoresearch Europe, Ely, United Kingdom). The negative staining was performed using a mixture of uranyl acetate/methylcellulose as previously described (Slot \& Geuze, 2007). Brightfield transmission electron micrographs of the stained EVs were taken at an indicated magnification of 40 000x using a JEM-2100Plus (JEOL, Herts, United Kingdom) TEM operating at an accelerating voltage of $80 \mathrm{kV}$, digital micrographs recorded using a Rio 16 CMOS camera using GMS 3.0 (Gatan, Pleasanton, CA, USA).

Total TG2 activity assay: TG2 activity of EVs either intact (resuspended in PBS) or lysed (resuspended in mild lysis buffer) was measured through incorporation of biotin cadaverine (BTC, Sigma-Aldrich) into FN as previously described (Jones, Nicholas et al., 1997). Samples were loaded in duplicates ( $N=3$ independent experiments). TG2 activity was calculated by removing the absorbance values obtained from the respective buffer (either PBS or lysis buffer) and expressed as $\mathrm{mU} / \mathrm{\mu g}$ of protein.

Cytoplasmic calcium imaging: Intracellular $\mathrm{Ca}^{2+}$ levels were assessed in hippocampal neurons loaded with Fura-2/AM dye (Merck, Darmstadt, Germany) as previously described (Joshi, Turola et al., 2014) and explained in the Appendix Extended methods. Neurons were distinguished from astrocytes by morphology and response to $\mathrm{KCl}$ stimuli (Bacci et al., 1999). Experiments were performed in Krebs-Ringer's HEPES solution (KRH) (125 mM $\mathrm{NaCl}, 5 \mathrm{mM} \mathrm{KCl}, 1.2 \mathrm{mM} \mathrm{MgSO}_{4}, 1.2 \mathrm{mM} \mathrm{KH}_{2} \mathrm{PO}_{4}, 2 \mathrm{mM} \mathrm{CaCl}_{2}, 6 \mathrm{mM}$ d-glucose, and $25 \mathrm{mM} \mathrm{HEPES} / \mathrm{NaOH}, \mathrm{pH}$ 7.4), whereas in some cases $\mathrm{Na}^{+} / \mathrm{K}^{+}$-free $\mathrm{KRH}$ was used instead, where both $\mathrm{NaCl}$ and $\mathrm{KCl}$ were isosmotically substituted for choline chloride. $\mathrm{Ca}^{2+}$ concentration was expressed as F340/380 fluorescence ratio as explained in the Appendix Extended methods. For each experiment, $\mathrm{N}$ indicates the total number of analysed neurons, either from multiple slides of the same cell preparation or from different preparations. Each neuronal preparation derived from $\geq 10$ rat $\mathrm{E} 18$ embryos.

Calcium imaging in brain slices: Slices were obtained from 10 days old CD1 mice and prepared as explained in the Appendix Extended methods. Acquisition protocols consisted of $1.2 \mathrm{fps}$ time-lapse sequences of Fura-2 
fluorescence with $30 \mathrm{~ms}$ exposure time. ROI over the field of view were selected, and the mean pixel intensity at each frame was measured. After 3 min baseline recording, $30 \mu \mathrm{g} / \mathrm{ml}$ TG2 (Zedira) in ACSF was perfused until plateau of the response was reached, then washed out. Analysis was performed using Metafluor software (Molecular Devices, San Jose, CA, USA).

Transient transfection: 8 DIV (days in vitro) neurons were transfected with $1.5 \mu \mathrm{g}$ of pEGFP-N1 vector (Clontech Laboratories, Takara Bio Inc., Mountain View, CA, USA) or pEGFP-N1-TG2 vector (Furini et al., 2018) using $6 \mu$ of Lipofectamine2000 (Thermo Fisher Scientific) in a total volume of $100 \mu$ l per slide (about $1.7 \times 10^{5}$ neurons). After 45 min incubation, transfected neurons were washed with Neurobasal medium and incubated in filtered conditioned neuronal medium for $48 \mathrm{~h}$ before analysis.

Whole cell patch clamp: Patch clamp experiments were performed in current clamp and voltage clamp configurations as explained in the Appendix Extended methods. For current clamp experiments, the effect of TG2 $(30 \mu \mathrm{g} / \mathrm{ml})$ on neuron resting potential was examined. Voltage clamp experiments were performed to observe the involvement of Voltage Operated Calcium Channels (VOCCs) in the phenomenon. Specifically, L-type VOCCs were isolated with a pre-pulse of $40 \mathrm{mV}$ and a $\Delta 10 \mathrm{mV}$ step protocol from -30 to $0 \mathrm{mV}$.

Isolation of TG2 complexes and Data Acquisition by Mass Spectrometry. TG2-associated proteins were isolated from hippocampal neurons incubated with $30 \mu \mathrm{g} / \mathrm{ml} \mathrm{TG2}$ in $\mathrm{KRH}$ buffer for $5 \mathrm{~min}$ and from untreated neurons as control $(\mathrm{N}=3)$. After incubation, the supernatant was removed and neurons were lysed using IP buffer (25 mM Tris-HCl, 150 mM NaCl, 1 mM EDTA, 1\% [v/v] NP40 detergent solution, and 5\% [v/v] glycerol, pH 7.4) containing protease inhibitor cocktail (Sigma-Aldrich). The whole cell lysate was centrifuged at $13000 \mathrm{xg}$ for 10 min at $4^{\circ} \mathrm{C}$ to remove larger particulates. TG2 with associated proteins was immunoprecipitated from cell lysates and supernatants as previously described (Furini et al., 2018) with few modifications. Incubation of samples with the anti-TG2 antibody-coated beads (CUB7402; Thermo Fisher Scientific) was performed for $20 \mathrm{~h}$ at $4^{\circ} \mathrm{C}$ in constant rotation. Proteins were reduced, alkylated and trypsin digested directly on the beads and peptides were analysed by RP-HPLC-ESI-MS/MS using a TripleTOF 6600 mass spectrometer (SCIEX) in data-dependent acquisition mode for spectral library construction, and in SWATH 2.0 data-independent acquisition mode using 100 variable SWATH acquisition windows for quantification as described in the Appendix Extended methods. The mass spectrometry proteomics data have been deposited to the ProteomeXchange Consortium via the PRIDE (Perez-Riverol, Csordas et al., 2019) partner repository with the dataset identifier "PXD022224". Analysis of 
differentially expressed proteins was performed using the OneOmics cloud processing online platform (SCIEX) as described in the Appendix Extended methods.

ATPase activity assay: $\mathrm{Na}^{+} / \mathrm{K}^{+}$-ATPase activity was analysed using the High Throughput Colorimetric ATPase Assay kit (Innova Biosciences, Cambridge, United Kingdom) according to the manufacturer's instructions. ATPase isolated from porcine cerebral cortex $(0.5 \mathrm{U} /$ well) was assayed in the presence or absence of gplTG2 (0.5 U/well) and specific $\mathrm{Na}^{+} / \mathrm{K}^{+}$-ATPase inhibitor ouabain $(1 \mathrm{mM})$.

Statistical analysis: All data are presented as mean \pm SD from the indicated number of independent experiments $(\mathrm{N}=3)$ or cells as indicated in the respective figure legends. Each primary cell preparation was derived from $\geq 10$ rat E18 embryos (hippocampal neurons) or $\geq 10$ mouse P3 pups (astrocytes). Data in each experiment were first tested for normal distribution by D’Agostino-Pearson normality test (GraphPad Prism 7.05 software). In the case of normally distributed data, statistical analysis was performed by Student's t-test (2 groups comparisons) or one-way Anova (Tukey multiple comparisons test). In the case of non-normally distributed data, statistical analysis was performed by nonparametric Mann-Whitney test (2 groups comparisons) or Kruskal-Wallis Dunn's test (multiple comparisons). For paired normalised data, the Wilcoxon matched-pairs signed rank test was used. Differences are considered significant by $p<0.05$.

Data Availability Statement: The mass spectrometry proteomics datasets have been deposited to the ProteomeXchange Consortium via the PRIDE (Perez-Riverol et al., 2019) partner repository with the dataset identifier "PXD022224" (http://www.ebi.ac.uk/pride). The rest of the data are included in this published article (and its supplementary information files). 


\section{Acknowledgments}

This work was supported by the John Turland PhD scholarship (to E.T. and E.A.M.V.), the American Association Research fellowship (to I.P. and C.V.) (AARF-588984), the IBRO/PERC InEurope Short Stay grant to E.T, the AIRC fellowship for Italy to I.V., The Marmont Foundation and the Nottingham Trent University REF-Quality Research fund (to E.A.M.V.). We are grateful to Prof Jeff Keillor (University of Ottawa, Canada) for critically reading the manuscript. We thank Prof David Abraham (University College London/Medical School, United Kingdom) and Prof Timothy Johnson (University of Sheffield, United Kingdom) for generously sharing facilities. We are grateful to Dr Giulia D'Arrigo (CNR Institute of Neuroscience, Italy) for her help in maintaining the primary cell cultures and we gratefully acknowledge the NTU Imaging Suite and Dr Graham J. Hickman for assistance in obtaining TEM data.

Author Contribution Statement: E.T., M.M, C.V. and E.A.M.V conceived and designed research. E.T., I.V., M.G., G.F., C.C. and M.P.S performed research. E.T., I.V., I.P., M.G., D.J.B., G.S., M.M., C.V. and E.A.M.V analysed and interpreted data. E.T., I.V., C.V. and E.A.M.V. wrote the paper. All authors read and approved the final paper.

Conflict of Interest Statement: The authors declare that they have no conflict of interest. 


\section{References}

Akimov SS, Krylov D, Fleischman LF, Belkin AM (2000) Tissue transglutaminase is an integrin-binding adhesion coreceptor for fibronectin. J Cell Biol 148: 825-38.

Andringa G, Lam KY, Chegary M, Wang X, Chase TN, Bennett MC (2004) Tissue transglutaminase catalyzes the formation of alpha-synuclein crosslinks in Parkinson's disease. FASEB J 18: 932-4.

Antonucci F, Turola E, Riganti L, Caleo M, Gabrielli M, Perrotta C, Novellino L, Clementi E, Giussani P, Viani P, Matteoli M, Verderio C (2012) Microvesicles released from microglia stimulate synaptic activity via enhanced sphingolipid metabolism. EMBO J 31: 1231-40.

Antonyak MA, Li B, Boroughs LK, Johnson JL, Druso JE, Bryant KL, Holowka DA, Cerione RA (2011) Cancer cell-derived microvesicles induce transformation by transferring tissue transglutaminase and fibronectin to recipient cells. Proc Natl Acad Sci U S A 108: 4852-7.

Bacci A, Verderio C, Pravettoni E, Matteoli M (1999) Synaptic and intrinsic mechanisms shape synchronous oscillations in hippocampal neurons in culture. Eur J Neurosci 11: 389-97.

Benilova I, Karran E, De Strooper B (2012) The toxic Abeta oligomer and Alzheimer's disease: an emperor in need of clothes. Nat Neurosci 15: 349-57.

Blaustein MP, Lederer WJ (1999) Sodium/calcium exchange: its physiological implications. Physiol Rev 79: 763854.

Carafoli E (1987) Intracellular calcium homeostasis. Annu Rev Biochem 56: 395-433.

Catterall WA, Few AP (2008) Calcium channel regulation and presynaptic plasticity. Neuron 59: 882-901.

Charrin S, Palmulli R, Billard M, Clay D, Boucheix C, Van Niel G, Rubinstein E (2020) Rapid Isolation of Rare Isotype-Switched Hybridoma Variants: Application to the Generation of IgG2a and IgG2b MAb to CD63, a Late Endosome and Exosome Marker. Antibodies (Basel) 9

Colak G, Johnson GV (2012) Complete transglutaminase 2 ablation results in reduced stroke volumes and astrocytes that exhibit increased survival in response to ischemia. Neurobiol Dis 45: 1042-50.

Colak G, Keillor JW, Johnson GV (2011) Cytosolic guanine nucledotide binding deficient form of transglutaminase 2 (R580a) potentiates cell death in oxygen glucose deprivation. PLoS One 6: e16665.

Colombo E, Farina C (2016) Astrocytes: Key Regulators of Neuroinflammation. Trends Immunol 37: 608-620. 
Condliffe SB, Corradini I, Pozzi D, Verderio C, Matteoli M (2010) Endogenous SNAP-25 regulates native voltagegated calcium channels in glutamatergic neurons. J Biol Chem 285: 24968-76.

D'Arrigo G, Gabrielli M, Scaroni F, Swuec P, Amin L, Pegoraro A, Adinolfi E, Di Virgilio F, Cojoc D, Legname G, Verderio C (2021) Astrocytes-derived extracellular vesicles in motion at the neuron surface: Involvement of the prion protein. J Extracell Vesicles 10: e12114.

De Laurenzi V, Melino G (2001) Gene disruption of tissue transglutaminase. Mol Cell Biol 21: 148-55.

Diaz-Hidalgo L, Altuntas S, Rossin F, D'Eletto M, Marsella C, Farrace MG, Falasca L, Antonioli M, Fimia GM, Piacentini M (2016) Transglutaminase type 2-dependent selective recruitment of proteins into exosomes under stressful cellular conditions. Biochim Biophys Acta 1863: 2084-92.

Farhy-Tselnicker I, Allen NJ (2018) Astrocytes, neurons, synapses: a tripartite view on cortical circuit development. Neural Dev 13: 7.

Feola J, Barton A, Akbar A, Keillor J, Johnson GVW (2017) Transglutaminase 2 modulation of NF-kappaB signaling in astrocytes is independent of its ability to mediate astrocytic viability in ischemic injury. Brain Res 1668: 1-11.

Forti L, Pouzat C, Llano I (2000) Action potential-evoked Ca2+ signals and calcium channels in axons of developing rat cerebellar interneurones. J Physiol 527 Pt 1: 33-48.

Furini G, Schroeder N, Huang L, Boocock D, Scarpellini A, Coveney C, Tonoli E, Ramaswamy R, Ball G, Verderio C, Johnson TS, Verderio EAM (2018) Proteomic Profiling Reveals the Transglutaminase-2 Externalization Pathway in Kidneys after Unilateral Ureteric Obstruction. J Am Soc Nephrol 29: 880-905.

Gabrielli M, Battista N, Riganti L, Prada I, Antonucci F, Cantone L, Matteoli M, Maccarrone M, Verderio C (2015) Active endocannabinoids are secreted on extracellular membrane vesicles. EMBO Rep 16: 213-20.

Goetzl EJ, Schwartz JB, Abner EL, Jicha GA, Kapogiannis D (2018) High complement levels in astrocyte-derived exosomes of Alzheimer disease. Ann Neurol 83: 544-552.

Grosso H, Mouradian MM (2012) Transglutaminase 2: biology, relevance to neurodegenerative diseases and therapeutic implications. Pharmacol Ther 133: 392-410.

Grosso H, Woo JM, Lee KW, Im JY, Masliah E, Junn E, Mouradian MM (2014) Transglutaminase 2 exacerbates alpha-synuclein toxicity in mice and yeast. FASEB J 28: 4280-91. 
Grover LM, Teyler TJ (1990) Two components of long-term potentiation induced by different patterns of afferent activation. Nature 347: 477-9.

Gylys KH, Fein JA, Cole GM (2000) Quantitative characterization of crude synaptosomal fraction (P-2) components by flow cytometry. J Neurosci Res 61: 186-92.

Hartley DM, Zhao C, Speier AC, Woodard GA, Li S, Li Z, Walz T (2008) Transglutaminase induces protofibril-like amyloid beta-protein assemblies that are protease-resistant and inhibit long-term potentiation. $J$ Biol Chem 283: 16790-800.

Holm MM, Kaiser J, Schwab ME (2018) Extracellular Vesicles: Multimodal Envoys in Neural Maintenance and Repair. Trends Neurosci 41: 360-372.

Hostenbach S, Cambron M, D'Haeseleer M, Kooijman R, De Keyser J (2014) Astrocyte loss and astrogliosis in neuroinflammatory disorders. Neurosci Lett 565: 39-41.

lentile R, Curro M, Caccamo D (2015) Transglutaminase 2 and neuroinflammation. Amino Acids 47: 19-26.

Jaggi AS, Singh N (2011) Therapeutic targets for the management of peripheral nerve injury-induced neuropathic pain. CNS Neurol Disord Drug Targets 10: 589-609.

Jones HC, Keep RF (1988) Brain fluid calcium concentration and response to acute hypercalcaemia during development in the rat. $J$ Physiol 402: 579-93.

Jones RA, Nicholas B, Mian S, Davies PJ, Griffin M (1997) Reduced expression of tissue transglutaminase in a human endothelial cell line leads to changes in cell spreading, cell adhesion and reduced polymerisation of fibronectin. J Cell Sci 110 ( Pt 19): 2461-72.

Joshi P, Turola E, Ruiz A, Bergami A, Libera DD, Benussi L, Giussani P, Magnani G, Comi G, Legname G, Ghidoni R, Furlan R, Matteoli M, Verderio C (2014) Microglia convert aggregated amyloid-beta into neurotoxic forms through the shedding of microvesicles. Cell Death Differ 21: 582-93.

Junn E, Ronchetti RD, Quezado MM, Kim SY, Mouradian MM (2003) Tissue transglutaminase-induced aggregation of alpha-synuclein: Implications for Lewy body formation in Parkinson's disease and dementia with Lewy bodies. Proc Natl Acad Sci U S A 100: 2047-52.

Katt WP, Antonyak MA, Cerione RA (2018) Opening up about Tissue Transglutaminase: When Conformation Matters More than Enzymatic Activity. Med One 3 
LaFerla FM (2002) Calcium dyshomeostasis and intracellular signalling in Alzheimer's disease. Nat Rev Neurosci 3: 862-72.

Leitch B, Szostek A, Lin R, Shevtsova O (2009) Subcellular distribution of L-type calcium channel subtypes in rat hippocampal neurons. Neuroscience 164: 641-57.

Marambaud P, Dreses-Werringloer U, Vingtdeux V (2009) Calcium signaling in neurodegeneration. Mol Neurodegener 4: 20.

Matsuda T, Arakawa N, Takuma K, Kishida Y, Kawasaki Y, Sakaue M, Takahashi K, Takahashi T, Suzuki T, Ota T, Hamano-Takahashi A, Onishi M, Tanaka Y, Kameo K, Baba A (2001) SEA0400, a novel and selective inhibitor of the $\mathrm{Na}+\mathrm{Ca} 2+$ exchanger, attenuates reperfusion injury in the in vitro and in vivo cerebral ischemic models. $J$ Pharmacol Exp Ther 298: 249-56.

Monteagudo A, Feola J, Natola H, Ji C, Proschel C, Johnson GVW (2018) Depletion of astrocytic transglutaminase 2 improves injury outcomes. Mol Cell Neurosci 92: 128-136.

Muma NA (2018) Transglutaminase in Receptor and Neurotransmitter-Regulated Functions. Med One 3

Nikoletopoulou V, Tavernarakis N (2012) Calcium homeostasis in aging neurons. Front Genet 3: 200.

Nimmrich V, Eckert A (2013) Calcium channel blockers and dementia. Br J Pharmacol 169: 1203-10.

Oh MM, Oliveira FA, Waters J, Disterhoft JF (2013) Altered calcium metabolism in aging CA1 hippocampal pyramidal neurons. J Neurosci 33: 7905-11.

Papouin T, Dunphy J, Tolman M, Foley JC, Haydon PG (2017) Astrocytic control of synaptic function. Philos Trans R Soc Lond B Biol Sci 372

Perez-Riverol Y, Csordas A, Bai J, Bernal-Llinares M, Hewapathirana S, Kundu DJ, Inuganti A, Griss J, Mayer G, Eisenacher M, Perez E, Uszkoreit J, Pfeuffer J, Sachsenberg T, Yilmaz S, Tiwary S, Cox J, Audain E, Walzer M, Jarnuczak AF et al. (2019) The PRIDE database and related tools and resources in 2019: improving support for quantification data. Nucleic Acids Res 47: D442-D450.

Pinzon NE, Breve JJP, Bol J, Drukarch B, Baron W, van Dam AM (2017) Tissue transglutaminase in astrocytes is enhanced by inflammatory mediators and is involved in the formation of fibronectin fibril-like structures. $J$ Neuroinflammation 14: 260.

Prada I, Gabrielli M, Turola E, lorio A, D'Arrigo G, Parolisi R, De Luca M, Pacifici M, Bastoni M, Lombardi M, Legname G, Cojoc D, Buffo A, Furlan R, Peruzzi F, Verderio C (2018) Glia-to-neuron transfer of miRNAs via 
extracellular vesicles: a new mechanism underlying inflammation-induced synaptic alterations. Acta Neuropathol 135: 529-550.

Pravettoni E, Bacci A, Coco S, Forbicini P, Matteoli M, Verderio C (2000) Different localizations and functions of L-type and N-type calcium channels during development of hippocampal neurons. Dev Biol 227: 581-94.

Quinn BR, Yunes-Medina L, Johnson GVW (2018) Transglutaminase 2: Friend or foe? The discordant role in neurons and astrocytes. J Neurosci Res 96: 1150-1158.

Raymond CR, Redman SJ (2002) Different calcium sources are narrowly tuned to the induction of different forms of LTP. J Neurophysiol 88: 249-55.

Scarpellini A, Huang L, Burhan I, Schroeder N, Funck M, Johnson TS, Verderio EA (2014) Syndecan-4 knockout leads to reduced extracellular transglutaminase-2 and protects against tubulointerstitial fibrosis. J Am Soc Nephrol 25: 1013-27.

Secondo A, Pignataro G, Ambrosino P, Pannaccione A, Molinaro P, Boscia F, Cantile M, Cuomo O, Esposito A, Sisalli MJ, Scorziello A, Guida N, Anzilotti S, Fiorino F, Severino B, Santagada V, Caliendo G, Di Renzo G, Annunziato L (2015) Pharmacological characterization of the newly synthesized 5-amino-N-butyl-2-(4ethoxyphenoxy)-benzamide hydrochloride (BED) as a potent NCX3 inhibitor that worsens anoxic injury in cortical neurons, organotypic hippocampal cultures, and ischemic brain. ACS Chem Neurosci 6: 1361-70.

Singh G, Zhang J, Ma Y, Cerione RA, Antonyak MA (2016) The Different Conformational States of Tissue Transglutaminase Have Opposing Affects on Cell Viability. J Biol Chem 291: 9119-32.

Slot JW, Geuze HJ (2007) Cryosectioning and immunolabeling. Nat Protoc 2: 2480-91.

Stellwagen D, Malenka RC (2006) Synaptic scaling mediated by glial TNF-alpha. Nature 440: 1054-9.

Stephens P, Grenard P, Aeschlimann P, Langley M, Blain E, Errington R, Kipling D, Thomas D, Aeschlimann D (2004) Crosslinking and G-protein functions of transglutaminase 2 contribute differentially to fibroblast wound healing responses. J Cell Sci 117: 3389-403.

Steppan J, Bergman Y, Viegas K, Armstrong D, Tan S, Wang H, Melucci S, Hori D, Park SY, Barreto SF, Isak A, Jandu S, Flavahan N, Butlin M, An SS, Avolio A, Berkowitz DE, Halushka MK, Santhanam L (2017) Tissue Transglutaminase Modulates Vascular Stiffness and Function Through Crosslinking-Dependent and CrosslinkingIndependent Functions. J Am Heart Assoc 6 
Tucholski J, Roth KA, Johnson GV (2006) Tissue transglutaminase overexpression in the brain potentiates calcium-induced hippocampal damage. J Neurochem 97: 582-94.

Turola E, Furlan R, Bianco F, Matteoli M, Verderio C (2012) Microglial microvesicle secretion and intercellular signaling. Front Physio/ 3: 149.

Verderio C, Bacci A, Coco S, Pravettoni E, Fumagalli G, Matteoli M (1999) Astrocytes are required for the oscillatory activity in cultured hippocampal neurons. Eur J Neurosci 11: 2793-800.

Verderio EA, Furini G, Burhan IW, Johnson TS (2015) Transglutaminases: Expression in kidney and relation to kidney fibrosis. In Transglutaminases Hitomi K, Kojima S, Fesus L (eds) pp 229-262. Springer Japan

Verderio EA, Telci D, Okoye A, Melino G, Griffin M (2003) A novel RGD-independent cel adhesion pathway mediated by fibronectin-bound tissue transglutaminase rescues cells from anoikis. J Biol Chem 278: 42604-14.

You Y, Borgmann K, Edara VV, Stacy S, Ghorpade A, Ikezu T (2020) Activated human astrocyte-derived extracellular vesicles modulate neuronal uptake, differentiation and firing. J Extracell Vesicles 9: 1706801. 


\section{Figure legends}

Figure 1. TG2 localises extracellularly in primary astrocytes and at synaptic sites in neurons. A) Immunofluorescence staining of primary astrocytes. Cells were fixed in $4 \%$ paraformaldehyde $-4 \%$ sucrose (w/v), permeabilized (left panels) or non-permeabilized (right panel) and stained with anti-TG2 IA12 (green), DAPI (blue) and astrocytic marker anti-GFAP (red). Coverslips were visualised by laser scanning Leica SP5 confocal microscope using $63 \mathrm{X}$ oil immersion objective. Successive serial optical sections $(1 \mu \mathrm{m})$ were recorded over $8 \mu \mathrm{m}$ planes. Scale bar $20 \mu \mathrm{m}$. TG2 intensity was calculated by Leica software, divided by number of nuclei, and normalised to permeabilized values. Data is expressed as mean $\pm S D(N=3$, Mann-Whitney test: $p=N S)$. B) Neurons at 12 DIV were fixed and permeabilized (left panel) or non-permeabilized (right panel) and stained with anti-TG2 IA12 (green), anti- $\beta$-TUB or anti-NR2B (red) antibodies and DAPI (blue). Scale bar $10 \mu \mathrm{m}$. TG2 intensity was calculated as described in $\mathrm{A}\left(\mathrm{N}=3\right.$, Mann-Whitney test: $\left.{ }^{\star} \mathrm{p}<0.05\right)$. C) Neurons at 22 Days In Vitro (DIV) were fixed, permeabilized and stained with anti-TG2 IA12 (green), anti-SHANK2 (red) and anti-VGLUT1 (blue) antibodies $(\mathrm{N}=3)$. Coverslips were visualised as described in A. Scale bar $20 \mu \mathrm{m}$ (low magnification) and $5 \mu \mathrm{m}$ (high magnification). D) Crude synaptosomes were isolated from adult mouse brain and probed with pre- and post-synaptic markers and for astrocyte marker GFAP. B-tubulin was used as loading control. TG2 is enriched in the synaptic fraction (Syn) compared to Low Speed Supernatant (LSS) representing the total lysate. Data is expressed as mean $\pm \mathrm{SD}\left(\mathrm{N}=4\right.$ independent experiments, Mann-Whitney test: $\left.{ }^{*} \mathrm{p}<0.05\right)$.

\section{Figure 2. Astrocytic TG2 is released in association with EVs and modulates calcium homeostasis in} neurons. A) EVs characterisation by nanoparticle tracking analysis (NTA). Average particle concentration (I) and mode size (II) of EVs isolated from untreated and LPS-treated WT and TG2KO astrocytes characterised by NTA (ZetaView, Particle Metrix). Data is shown as mean $\pm \mathrm{SD}(\mathrm{N}=3$ independent experiments, Kruskal-Wallis Dunn's test: $p=N S$ ). (III) Graph showing average particle concentration according to EVs size (Kruskal-Wallis Dunn's test WT untreated - WT LPS: *p<0.05). B) EVs characterisation by Transmission electron microscopy (TEM). TEM analysis of EVs isolated from untreated WT astrocytes and stained with either anti-CD63 antibody (Abcam) or anti-TG2 antibody (IA12) and $6 \mathrm{~nm}$ gold colloidal anti-mouse IgG (top panels), or negative stained with uranyl acetate methylcellulose (bottom panel). C) Western blotting analysis of astrocytes-derived EVs and proteins TCAprecipitated from vesicle-free supernatants (EV-free SN) for the detection of EVs markers ALIX and FLOT-2, EVs 
negative marker TOM20, glial marker GFAP and of TG2. $\beta$-tubulin was used as loading control for TL. A representative blot of 3 independent experiments is shown. Treated cells were primed with LPS ( $1 \mu \mathrm{g} / \mathrm{ml})$ for $24 \mathrm{~h}$ in serum-free medium before EVs isolation. Vertical black lines separate sections of the same membrane developed for different exposition times. D) Comparison of TG2 activity in intact and lysed EVs isolated from WT and TG2KO astrocytes. EVs were either resuspended as intact in PBS or lysed in a mild lysis buffer, to detect surface TG2 activity or total activity respectively. Data are expressed as mean \pm SD normalised to WT intact EVs values ( $\mathrm{N}=3$ independent experiments; Kruskal-Wallis Dunn's test: ${ }^{*} \mathrm{p}<0.05$ ). E) Quantification of $\left[\mathrm{Ca}^{2+}\right]_{\text {, }}$ expressed as F340/380, in 13 DIV neurons before and after stimulation with EVs isolated from LPS-treated WT or TG2KO astrocytes ( $\mathrm{N} \geq 75$ neurons from a preparation derived from $\geq 10$ rat $\mathrm{E} 18$ embryos). EVs from either WT or TG2KO cells were resuspended in $\mathrm{KRH}$ supplemented with $30 \mathrm{U} / \mathrm{ml}$ of apyrase and added to neurons in the presence of $1 \mu \mathrm{M} \mathrm{TTX}, 50 \mu \mathrm{M}$ APV and $10 \mu \mathrm{M}$ CNQX. $\left[\mathrm{Ca}^{2+}\right]_{\mathrm{i}}$ was measured in multiple fields before (basal) and 20-60 min after addition of either WT or TG2KO EVs. Data are expressed as mean \pm SD (one-way ANOVA test: $\left.{ }^{*} \mathrm{p}<0.05\right)$.

Figure 3. Exogenous TG2 increases interspike and basal $\left[\mathrm{Ca}^{2+}\right]_{i}$ in hippocampal neurons in culture and in brain slices. A) (I) Representative temporal analysis of synchronous $\mathrm{Ca}^{2+}$ oscillations, expressed as F340/380, spontaneously occurring in 14 DIV Fura-2-loaded hippocampal neurons. Addition of exogenous TG2 promoted a $\mathrm{Ca}^{2+}$ spike followed by a sustained $\mathrm{Ca}^{2+}$ plateau (indicated by $\Delta \mathrm{F} 340 / 380$ ), with a block of oscillations. Upon TG2 removal (wash with $\mathrm{KRH}$ ) synchronous $\mathrm{Ca}^{2+}$ oscillations re-started. Three representative traces of neuronal $\left[\mathrm{Ca}^{2+}\right]_{\mathrm{i}}$ are shown in each graph. The graph shows the quantification of TG2-induced interspike $\left[\mathrm{Ca}^{2+}\right]_{i}$ elevations compared with basal $\left[\mathrm{Ca}^{2+}\right]_{i}\left(\mathrm{~N}=23\right.$ neurons, Student's t-test: $\left.{ }^{* \star \star} \mathrm{p}<0.0001\right)$. (II) Addition of exogenous TG2 in the presence of TTX $(1 \mu \mathrm{M})$ significantly increased $\left[\mathrm{Ca}^{2+}\right]_{i}$ (indicated by $\left.\Delta \mathrm{F} 340 / 380\right)$. Graph shows the quantification of TG2-induced $\left[\mathrm{Ca}^{2+}\right]_{i}$ rises (peak $\mathrm{Ca}^{2+}$ rise) compared with basal $\left[\mathrm{Ca}^{2+}\right]_{i}$ in the presence of TTX $(\mathrm{N}=33$ neurons, Student's t-test: ${ }^{* * *} \mathrm{p}<0.0001$ ). Data are presented as mean F340/380 \pm SD. B) (I) Representative DIC (top) and $380 \mathrm{~nm}$ fluorescence (bottom) images of neurons in the stratum pyramidale (sp) of mouse hippocampal slices loaded with Fura-2/AM for calcium imaging experiments. The stratum oriens (so) and stratum radiatum (sr) are indicated above and below sp respectively. (II) Representative temporal plot of $\left[\mathrm{Ca}^{2+}\right]_{\mathrm{i}}$ rises evoked by perfusion of extracellular TG2 in fura-2 loaded hippocampal slices in $1 \mu \mathrm{M}$ TTX ( $N=3$ brain slices, 28 neurons in total). C) 
Temporal analysis of $\left[\mathrm{Ca}^{2+}\right]_{i}$ changes in 14-20 DIV neurons exposed to exogenous TG2 in the absence (I) or in the presence (II) of extracellular $\mathrm{Ca}^{2+}$. Addition of TG2 in the absence of extracellular $\mathrm{Ca}^{2+}$ did not lead to changes in $\left[\mathrm{Ca}^{2+}\right]_{\mathrm{i}}(\mathrm{N}=31$ neurons $)$.

Figure 4. Transfected TG2 induces a calcium rise in an autocrine manner in neurons. A) (I) Representative traces showing basal $\left[\mathrm{Ca}^{2+}\right]$, expressed as F340/380, in pEGFP-N1-TG2 or pEGFP-N1 transfected neurons (8 DIV). (II) Graph showing [ $\left.\mathrm{Ca}^{2+}\right]$, in transfected neurons expressed as mean F340/380 \pm SD normalised to EGFP $\left(\mathrm{N}>100\right.$ neurons. Mann-Whitney test: $\left.{ }^{* * *} \mathrm{p}<0.0001\right)$. B) A subgroup of transfected neurons was treated with the non-permeable TG2 inhibitor BOCDON $(200 \mu \mathrm{M})$, which did not affect $\left[\mathrm{Ca}^{2+}\right]_{i}(\mathrm{~N}>45$ neurons). A significant difference in $\left[\mathrm{Ca}^{2+}\right]_{i}$ between EGFP and EGFP-TG2 was observed in this subgroup as in graph A-II (one-way ANOVA test: $\left.{ }^{* *} p<0.01\right)$.

Figure 5. Exogenous TG2 mediates $\mathrm{Ca}^{2+}$ influx from the extracellular environment. A) Temporal analysis of $\left[\mathrm{Ca}^{2+}\right]_{\mathrm{i}}$ changes in 14-20 DIV neurons exposed to exogenous TG2 in the absence (I) or in the presence (II) of APV (NMDA receptor antagonist) and CNQX (AMPA/kainate receptor antagonist). TG2-dependent $\mathrm{Ca}^{2+}$ rise was not affected by the blockers. (III) Corresponding quantification of TG2-induced peak $\left[\mathrm{Ca}^{2+}\right]_{i}$ rises. Data are presented as mean $\triangle \mathrm{F} 340 / 380 \pm \mathrm{SD}(\mathrm{N}=46$ and 9 neurons for TG2 alone and TG2+blockers respectively; Mann-Whitney test: $\mathrm{p}=\mathrm{NS}$ ). B) Pre-treatment with cadmium (I), a general blocker of $\mathrm{Ca}^{2+}$ channels, and nickel (II), a blocker of Ttype VOCCs, reduced TG2-dependent $\mathrm{Ca}^{2+}$ responses of about $82.4 \%$ and $66.2 \%$ respectively, as quantified in (III), by measuring total $\mathrm{Ca}^{2+}$ influxes. Data are expressed as mean area under the curve (AUC) $\pm \mathrm{SD}$ of TG2 + blockers normalised to TG2 alone ( $\mathrm{N}=7$ and 15 neurons for treatment with $\mathrm{Cd}^{2+}$ and $\mathrm{Ni}^{2+}$ respectively Wilcoxon signed-rank test: ${ }^{\star} \mathrm{p}<0.05 ;{ }^{* \star \star *} \mathrm{p}<0.0001$ ). C) Addition of cadmium (I) and nickel (II) during the plateau phase of TG2-induced $\mathrm{Ca}^{2+}$ response led to a recovery of $\left[\mathrm{Ca}^{2+}\right]_{i}$ towards basal levels. (III) Histograms show residual mean $\mathrm{Ca}^{2+}$ responses after blockers normalised to peak $\mathrm{Ca}^{2+}$ response evoked by TG2 $\pm \mathrm{SD}(\mathrm{N}=18$ and 25 neurons for treatment with $\mathrm{Cd}^{2+}$ and $\mathrm{Ni}^{2+}$ respectively; Wilcoxon signed-rank test: $\left.{ }^{* * *} \mathrm{p}<0.0001\right)$. D) $\mathrm{Ca}^{2+}$ transients induced by $15 \mathrm{mM} \mathrm{KCl}$ in the presence of NMDAR and ampa/kainate receptor blockers APV and CNQX revealed that $\left[\mathrm{Ca}^{2+}\right]_{\mathrm{i}}$ rose faster and led to a 2-fold higher total $\mathrm{Ca}^{2+}$ influx in the presence of TG2. Data are expressed as mean AUC of $\mathrm{TG} 2+\mathrm{KCl}$ normalised to $\mathrm{KCl}$ alone treatment $\pm \mathrm{SD}\left(\mathrm{N}=22\right.$ neurons; Wilcoxon signed-rank test: $\left.{ }^{* \star *} \mathrm{p}<0.0001\right)$. 
E) Temporal analysis of $\left[\mathrm{Ca}^{2+}\right]_{i}$ changes induced by TG2 in the presence of L-type VOCCs blocker Nifedipine (NF). (I) Total $\mathrm{Ca}^{2+}$ influxes in response to TG2 were reduced of about $36 \%$ in neurons pre-treated with NF. Data are expressed as mean AUC $\pm \mathrm{SD}$ induced by $\mathrm{TG} 2$ in the presence of NF normalised to TG2 alone $(\mathrm{N}=21$ neurons; Wilcoxon signed-rank test: ${ }^{* * *} \mathrm{p}<0.0001$ ). (II) NF caused a partial recovery of about $63 \%$ of $\mathrm{Ca}^{2+}$ concentration towards resting levels when applied during the plateau phase of $\mathrm{Ca}^{2+}$ response induced by exogenous TG2. Histograms show mean residual $\mathrm{Ca}^{2+}$ responses $\pm \mathrm{SD}$ after NF normalised to peak $\mathrm{Ca}^{2+}$ response evoked by TG2 alone ( $N=25$ neurons; Wilcoxon signed-rank test: $\left.{ }^{* * * *} p<0.0001\right)$.

\section{Figure 6. TG2 activates an inward calcium current through L-type VOCC membrane depolarisation. A)}

Whole cell current clamp. (I) Addition of TG2 induced a slow membrane depolarisation of about $20 \mathrm{mV}$ ( $\mathrm{N}=3$ ). (II) Addition of Nickel after TG2 re-established resting membrane potential. B) Voltage clamp. TG2 perfusion promoted excitatory postsynaptic currents (EPSCs) consistent with the activation of an inward $\mathrm{Ca}^{2+}$ current $(\mathrm{N}=3)$. C) Whole cell voltage clamp results expressed as current/voltage (I/V) curves. (I) Perfusion of TG2 (red I/V curve) led to an increased inward current compared to control (black I/V curve). The graph on the right shows current recordings of the same experiment at $-20 \mathrm{mV}$. The protocol for isolation of L-type VOCCs was applied as described in the Methods. (II) In the presence of NF (blue I/V curve), TG2 perfusion (red I/V curve, left panel) did not increase inward current compared to control (black I/V curve). The graph on the right shows current recordings of the same experiment at $-20 \mathrm{mV}(\mathrm{N}=3)$.

Figure 7. Contribution of NCX to TG2-dependent calcium rise. Temporal analysis of $\left[\mathrm{Ca}^{2+}\right]_{i}$ changes in $14-20$ DIV neurons exposed to exogenous TG2. A) Addition of TG2 in $\mathrm{Na}^{+}$-free $\mathrm{KRH}$ led to an 8-fold increase in average $\mathrm{Ca}^{2+}$ influx compared to normal $\mathrm{KRH}\left(\mathrm{N}=20\right.$ neurons; Wilcoxon signed-rank test: $\left.{ }^{* \star * *} \mathrm{p}<0.0001\right)$. Data are expressed as mean \pm SD normalised to TG2 AUC values. B) Representative temporal analysis of neuronal $\left[\mathrm{Ca}^{2+}\right]_{\mathrm{i}}$ responses evoked by TG2 in the absence and in the presence of the NCX inhibitor YM-244769 (10 min) in $\mathrm{Na}^{+}-$ free KRH. YM-244769 induced a significant decrease in TG2-dependent $\mathrm{Ca}^{2+}$ influx. The graph shows mean TG2induced AUC \pm SD after YM-244769 normalised to TG2 alone in normal KRH ( $N=25$ neurons; Wilcoxon signedrank test: $\left.{ }^{* * *} \mathrm{p}<0.0001\right)$ and $\mathrm{Na}^{+}$-free $\mathrm{KRH}\left(\mathrm{N}=21\right.$ neurons; Wilcoxon signed-rank test: $\left.{ }^{* * \star *} \mathrm{p}<0.0001\right)$. 
Figure 8. TG2 interactors in the neuronal extracellular environment: inhibition of $\mathrm{Na}^{+} / \mathrm{K}^{+}$-ATPase. A) Workflow of the identification of TG2-interactors. TG2 was immunoprecipitated from hippocampal neurons (total lysate including the secretome) incubated with extracellular TG2 or untreated ( $\mathrm{N}=3$ independent immunoprecipitations, from a neuronal preparation derived from $\geq 10$ rat E18 embryos). TG2 co-precipitated proteins (TG2-IP) were trypsin digested on beads and analysed by SWATH MS. SWATH quantitative data were extracted using a spectral library produced by shotgun/data dependent acquisition (DDA) MS on all TG2-IP samples. B) Selection of TG2 interactors shown according to logarithm of fold change $\left(\log _{2} \mathrm{FC}\right)$ between neurons exposed to extracellular TG2 and untreated cells. C) The activity of porcine cerebral cortex ATPase (0.5 mU/well) assayed in the absence or presence of either gpITG2 (0.5 mU/well, $43.9 \mathrm{nM})$ or Ouabain ( $1 \mathrm{mM})$. Data are shown as mean $\pm \mathrm{SD}$ of ATPase activity $(\mathrm{mU} / \mathrm{ml})$, normalised to ATPase alone values ( $\mathrm{N}=3$ independent experiments, one-way ANOVA test: $\left.{ }^{*} p<0.05 ;{ }^{* * * *} p<0.0001\right)$.

Figure 9. Schematic representation of the proposed mechanism. A) Activated astrocytes release EVs containing TG2 as a cargo and displayed at the EVs surface. B) Extracellular TG2 (either astrocytes vesiclebound or free in the medium) interacts with neurons and increases $\left[\mathrm{Ca}^{2+}\right]_{i}$ via (C) inhibition of $\mathrm{Na}^{+} / \mathrm{K}^{+}-\mathrm{ATPase}$, causing membrane depolarisation (about $20 \mathrm{mV}$ ) and activation of an inward $\mathrm{Ca}^{2+}$ current through (D) L-type VOCCs and (E) the $\mathrm{Na}^{+} / \mathrm{Ca}^{2+}$ exchanger (NCX) leading to (F) increase of $\left[\mathrm{Ca}^{2+}\right]_{i}$ and $\mathrm{Ca}^{2+}$ dyshomeostasis. 


\section{Expanded View Figure Legends}

Figure EV1. TG2 is localised on the surface of primary astrocytes. Cell surface TG2 activity in primary astrocytes (up to $44 \mathrm{DIV})$ using TG2 inhibitor ZDON (100 $\mu \mathrm{M})$ to validate TG2 specific activity. Data are shown as mean \pm SD of raw absorbance values minus background, normalised to CTR in the presence of $0.1 \%$ DMSO (vehicle) $\left(\mathrm{N}=4\right.$, Mann-Whitney test: $\left.{ }^{* \star *} \mathrm{p}<0.001\right)$.

Figure EV2. TG2 localises extracellularly in primary astrocytes and co-localises with fibronectin. Immunofluorescence staining of primary astrocytes. Cells were fixed in $3 \%$ paraformaldehyde $(\mathrm{w} / \mathrm{v})$ without permeabilization and stained with anti-TG2 IA12 (green), DAPI (blue) and ECM marker fibronectin (FN, red). Coverslips were visualised by laser scanning Leica SP5 confocal microscope using 63X oil immersion objective. Successive serial optical sections $(1 \mu \mathrm{m})$ were recorded over $8 \mu \mathrm{m}$ planes. Scale bar $20 \mu \mathrm{m}$.

Figure EV3. TG2 in KRH has negligible activity in the absence of reducing agent (DTT). Total TG activity assay assessing TG2 activity levels in vitro expressed as $\mathrm{mU} / \mu \mathrm{g}$ of protein. TG2 was incubated in $\mathrm{KRH}$ buffer $\mathrm{pH}$ 7.4 (which contains $2 \mathrm{mM}$ calcium) in the presence or absence of DTT $10 \mathrm{mM}$. Data is expressed as mean \pm SD $\left(\mathrm{N}=3\right.$, each point analysed in duplicate. Mann-Whitney test: $\left.{ }^{* *} \mathrm{p}<0.01\right)$. 
Figure 1

A

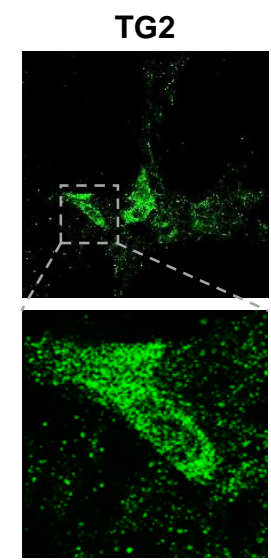

Permeabilized astrocytes

B

Permeabilized neurons

TG2

$\beta$-TUB

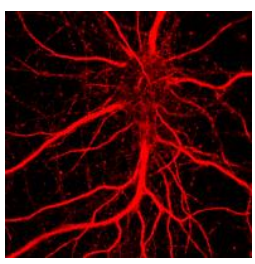

C
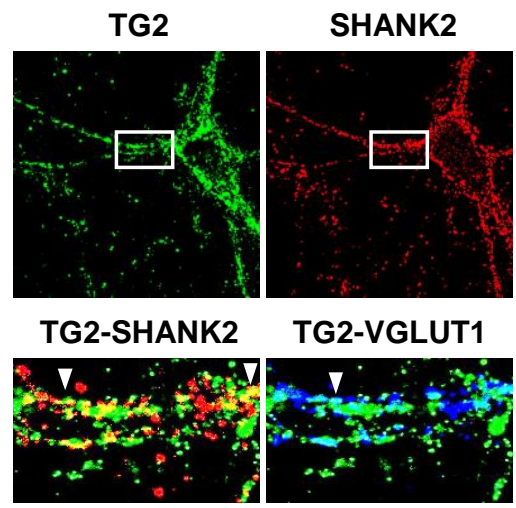

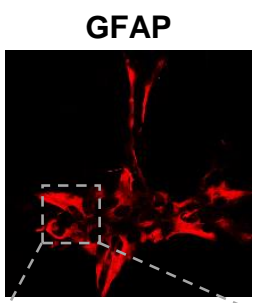

Total merge
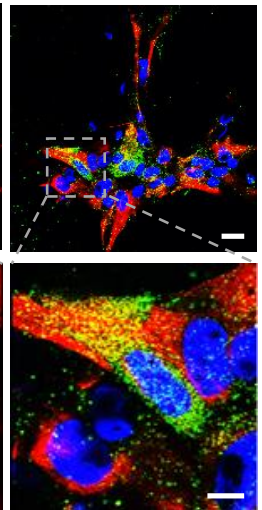

Non-permeabilized astrocytes
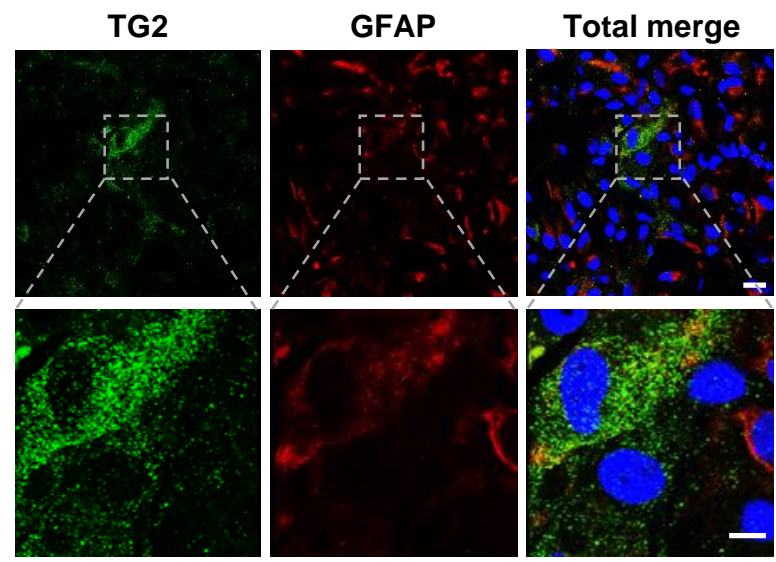

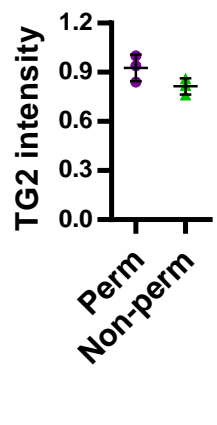

Non-permeabilized neurons

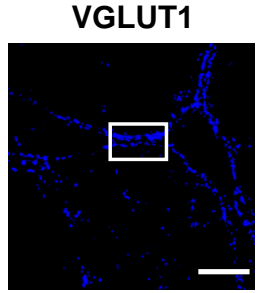

TG2

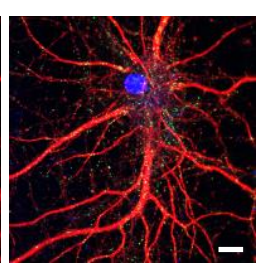

D

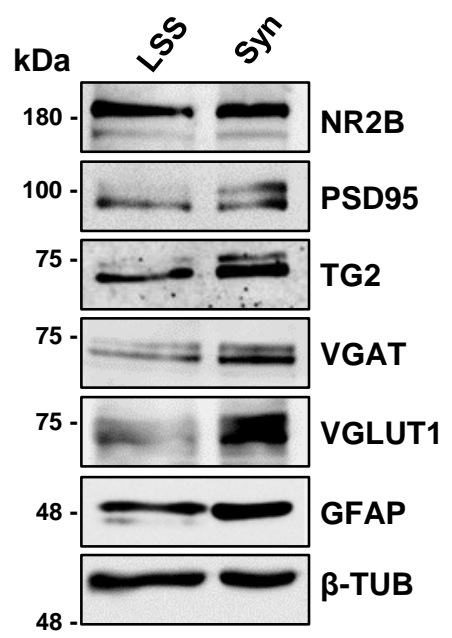

Merge

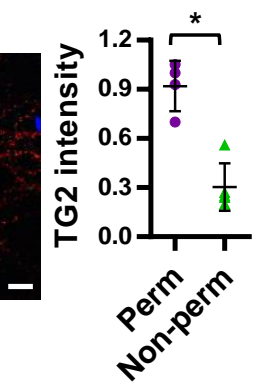


Figure 2

A

(I)

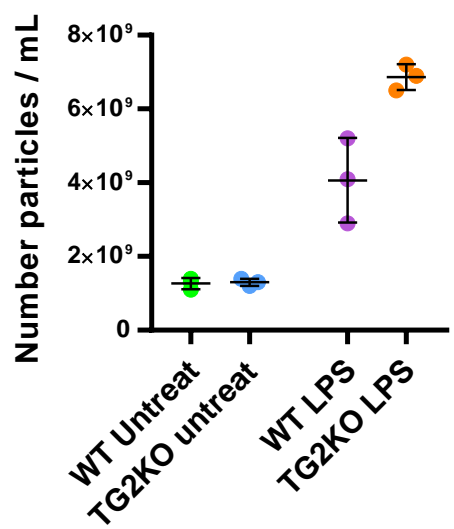

B

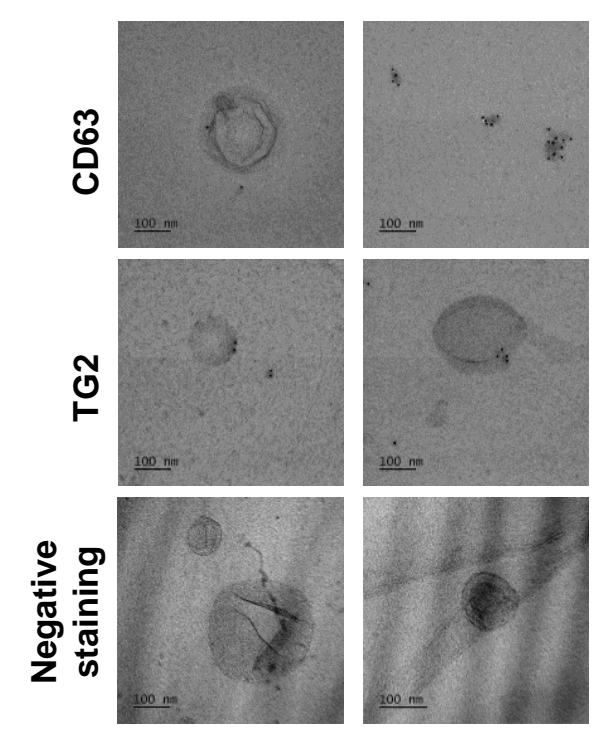

D

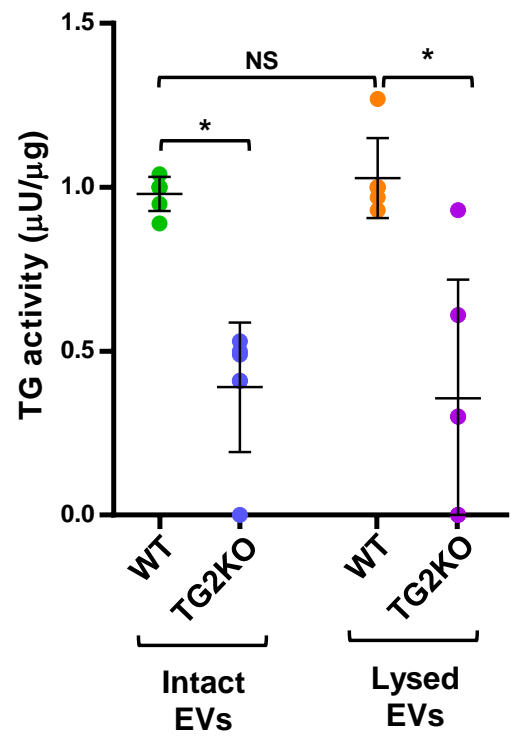

(II)

(III)

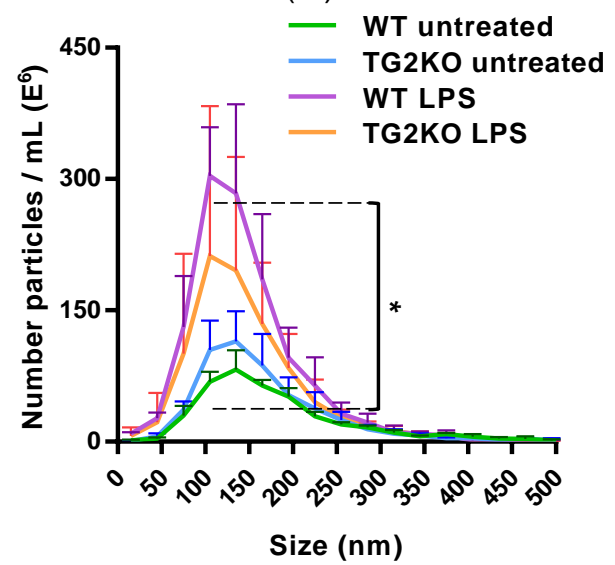

C

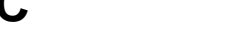

WT

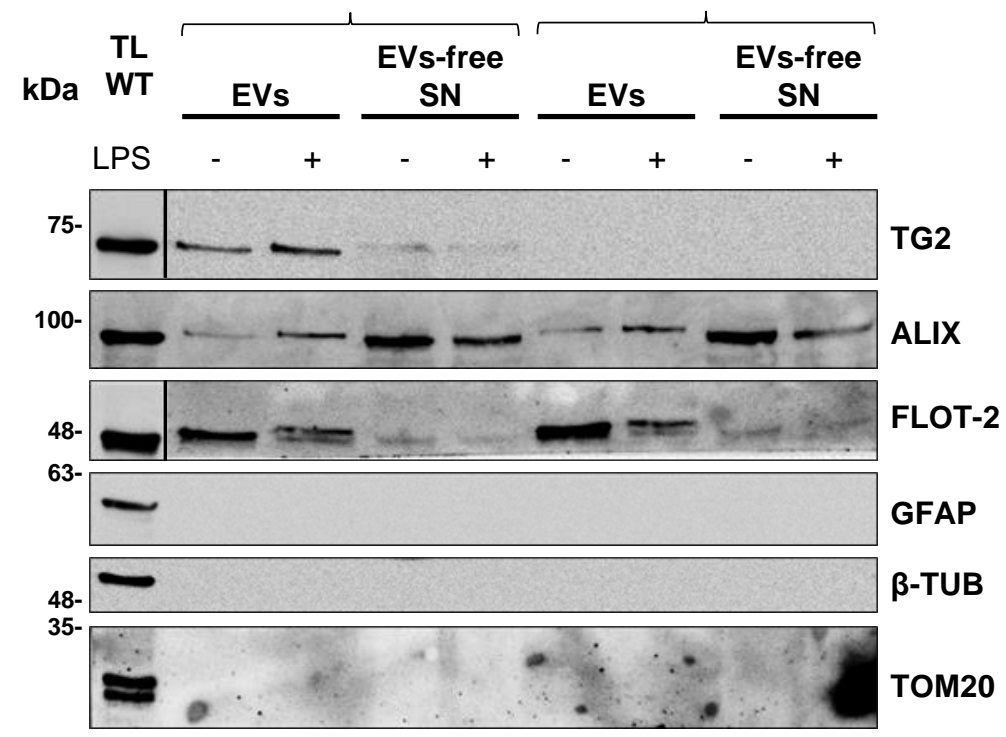

E

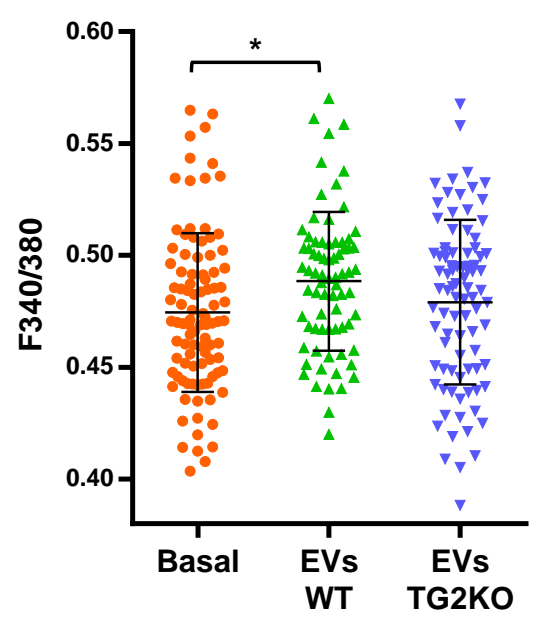


Figure 3
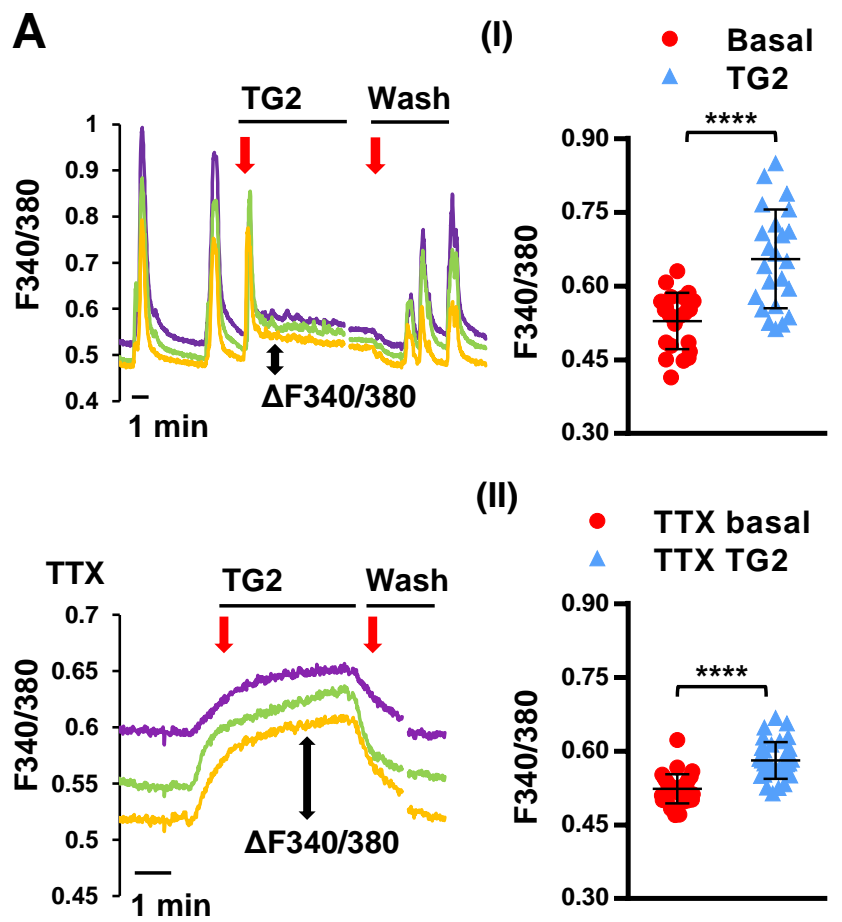

B

Hippocampal slices

(I)

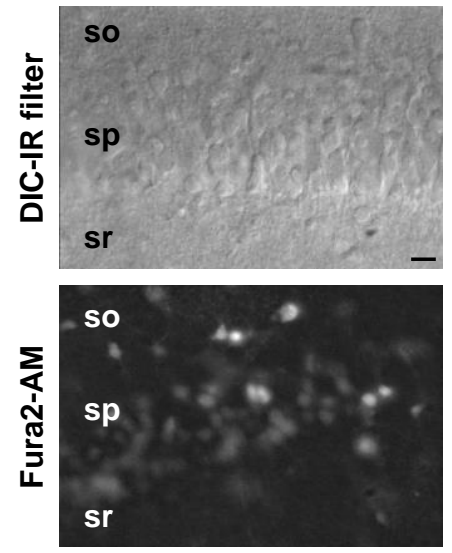

C
(II)

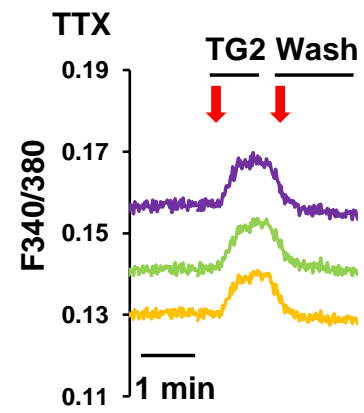

(II)

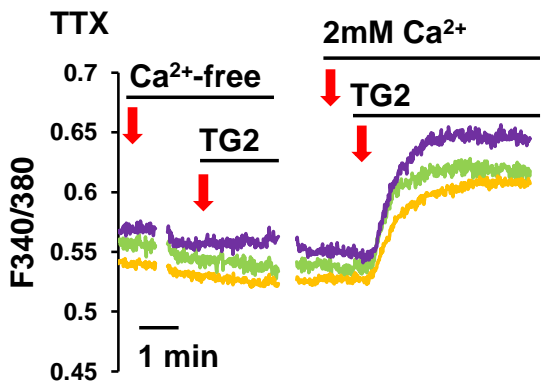


Figure 4

A

(I) EGFP-1 EGFP-2 EGFP-3
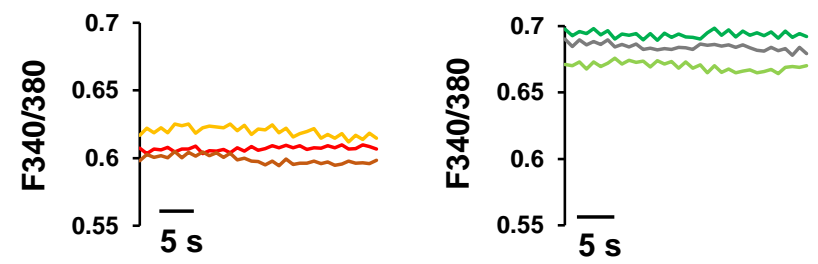

(II)

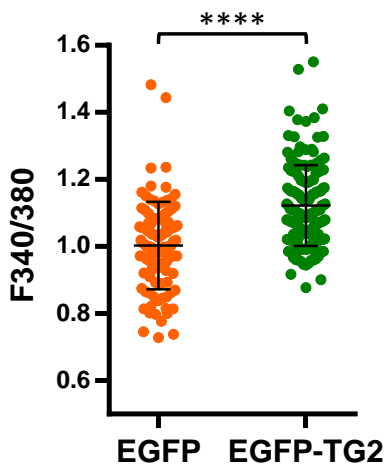

B

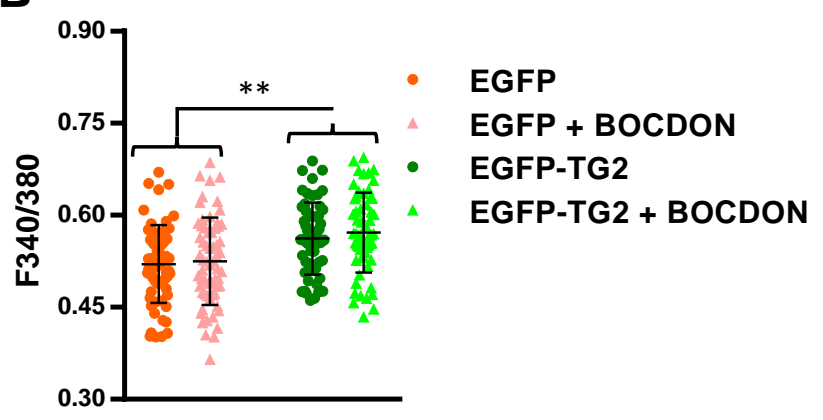




\section{Figure 5}

A

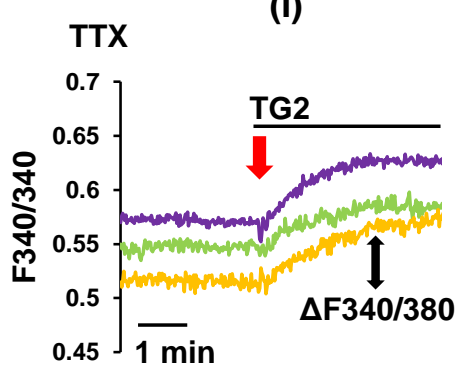

B

TTX

(I)

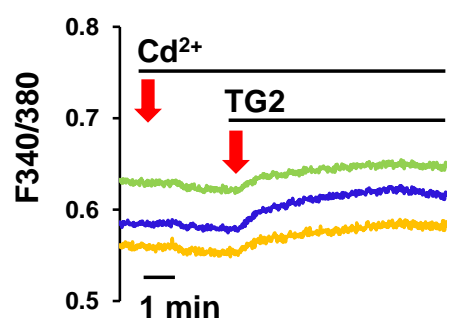

C

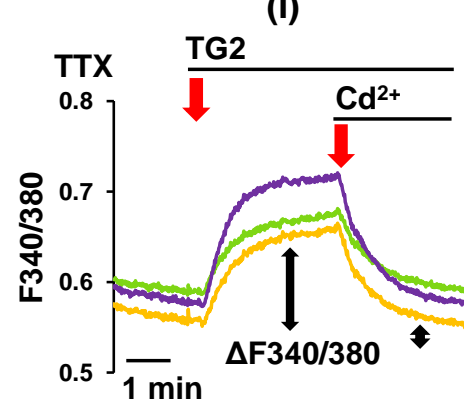

D

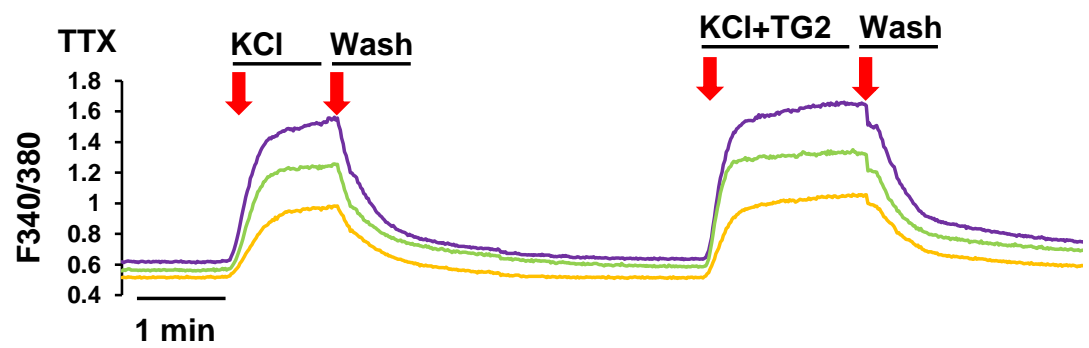

E

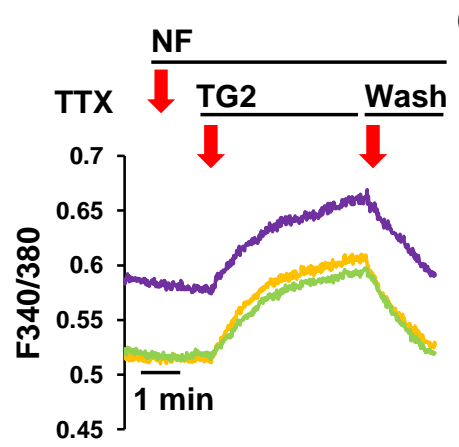

(I)

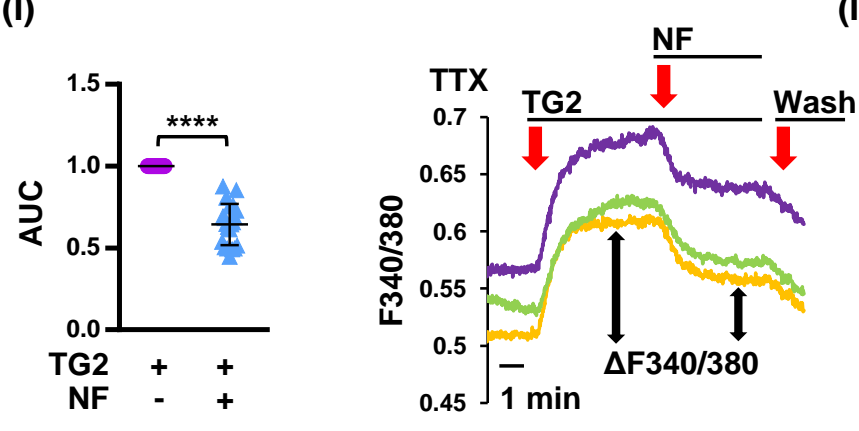

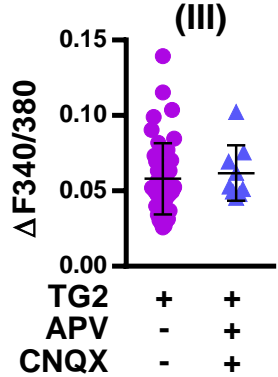

(II)

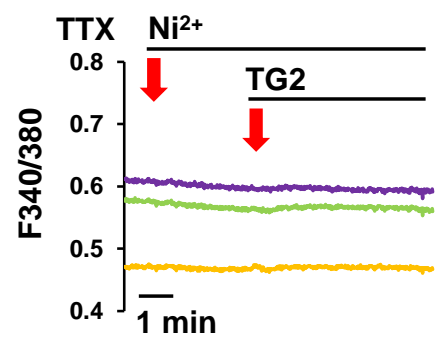

(II)
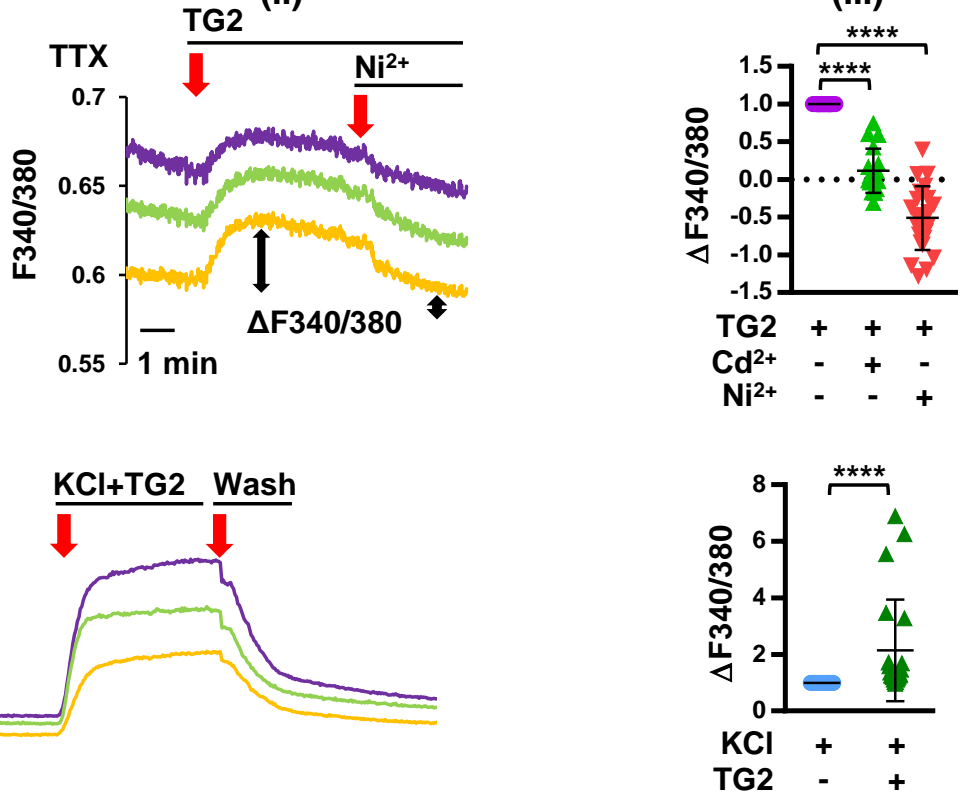

(II)

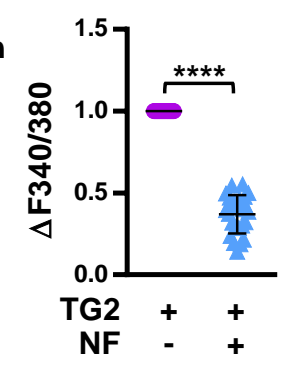


Figure 6

A

(I)

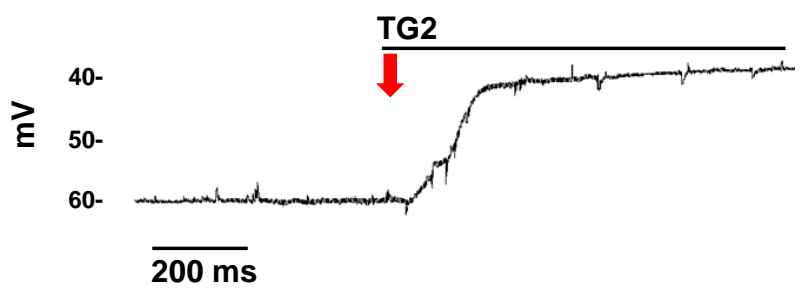

(II)

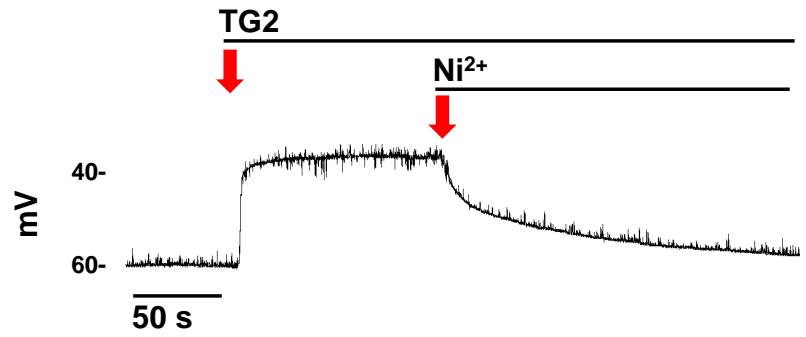

B

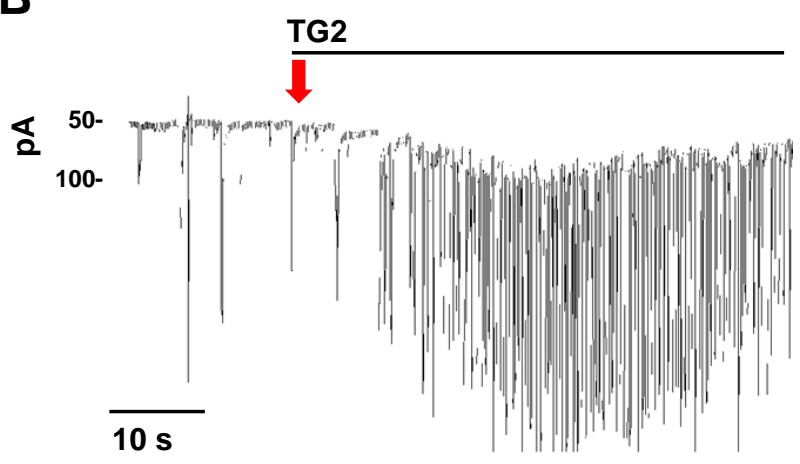

C

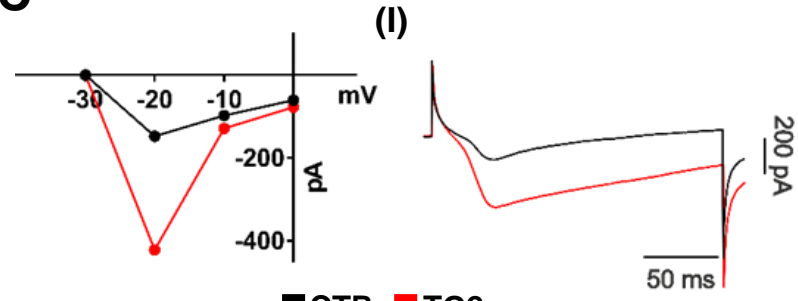

(II)

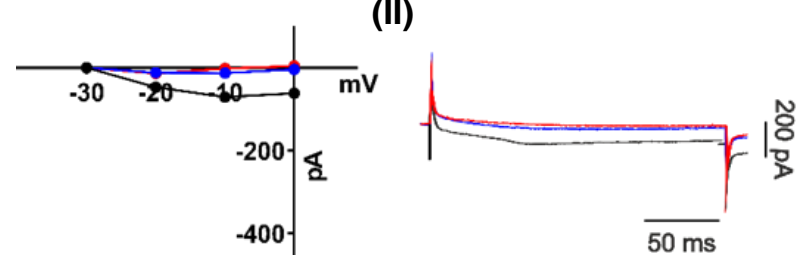

ICTR $\square$ NF $\square$ TG2+NF 
Figure 7

A
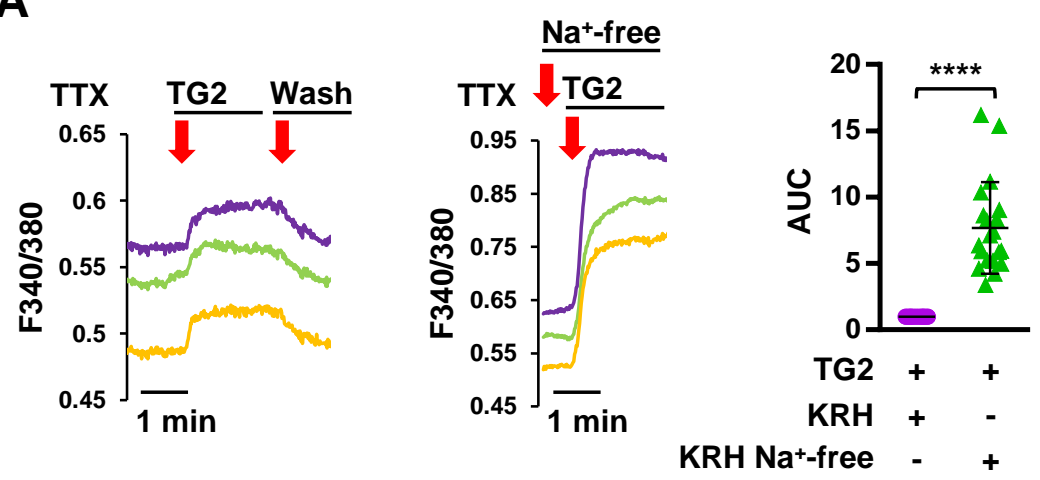

B

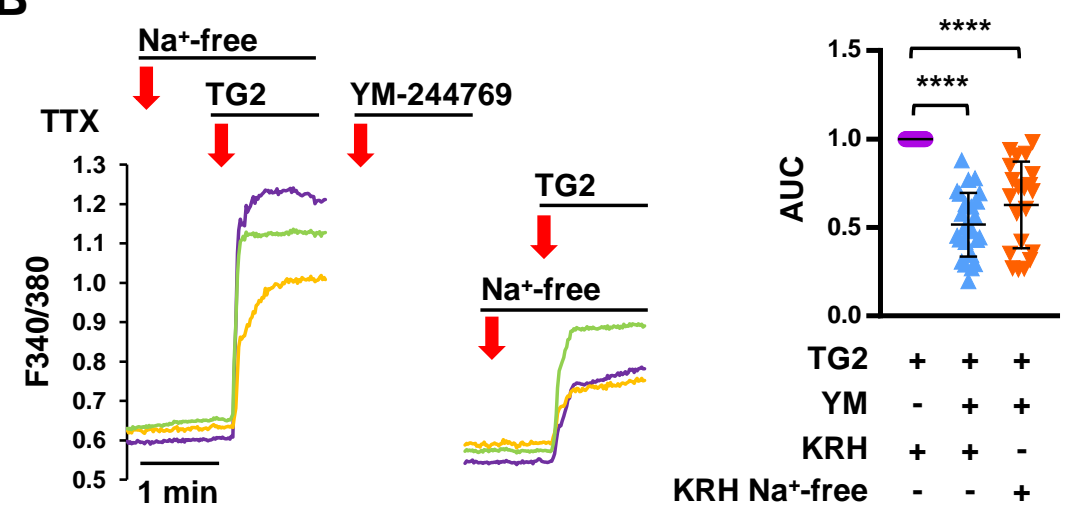


B
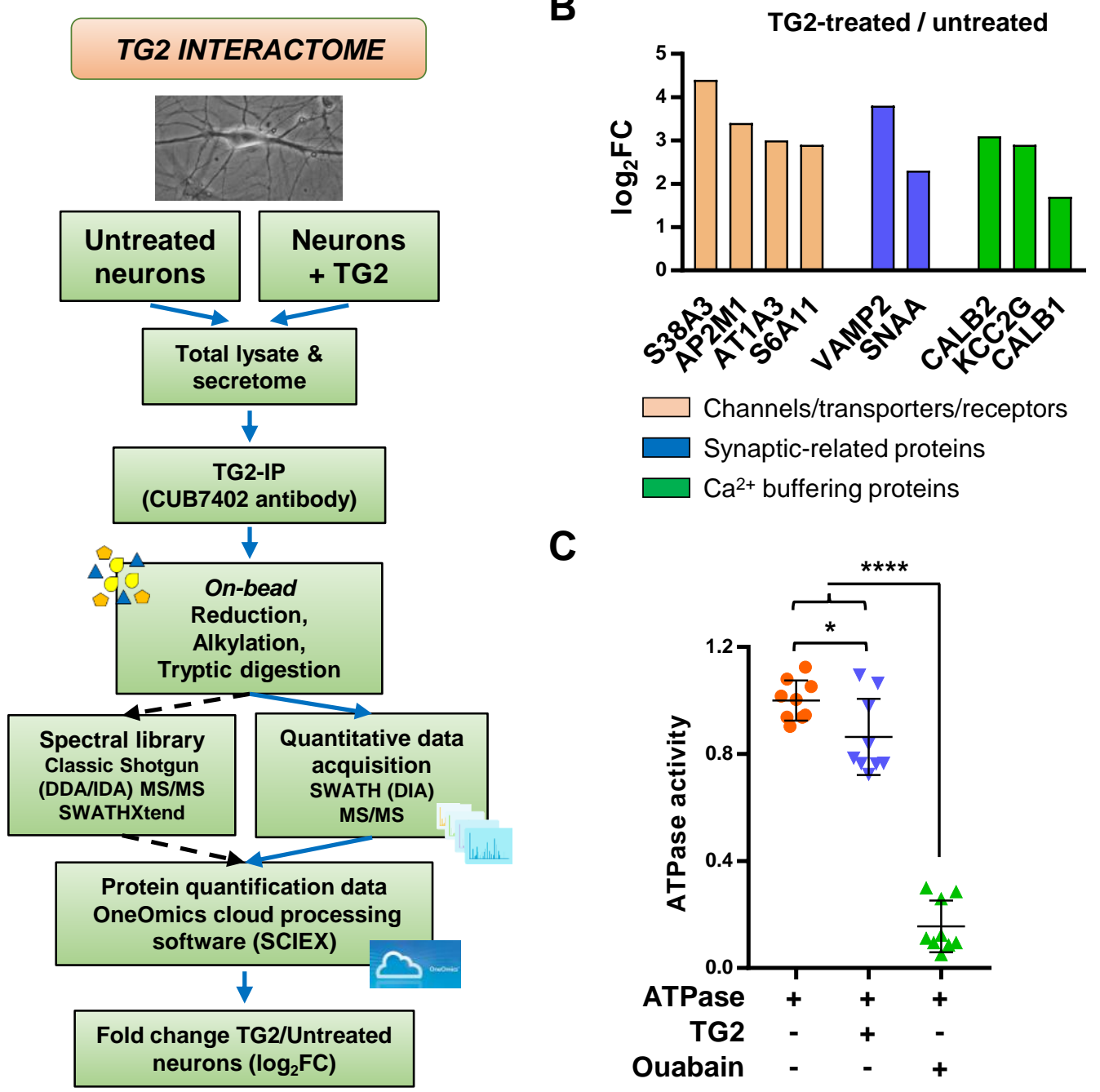

C

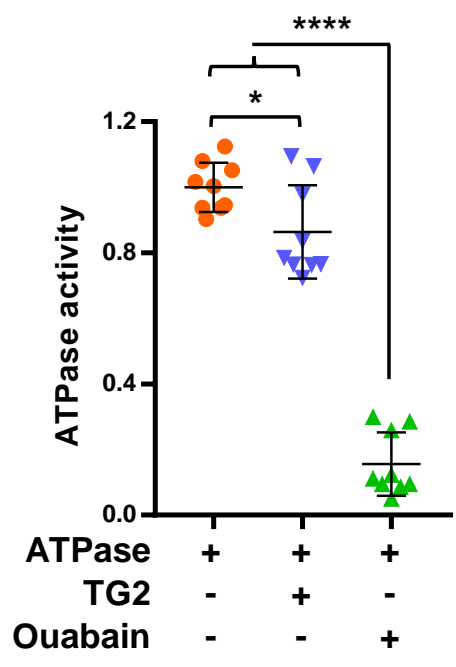


Figure 9

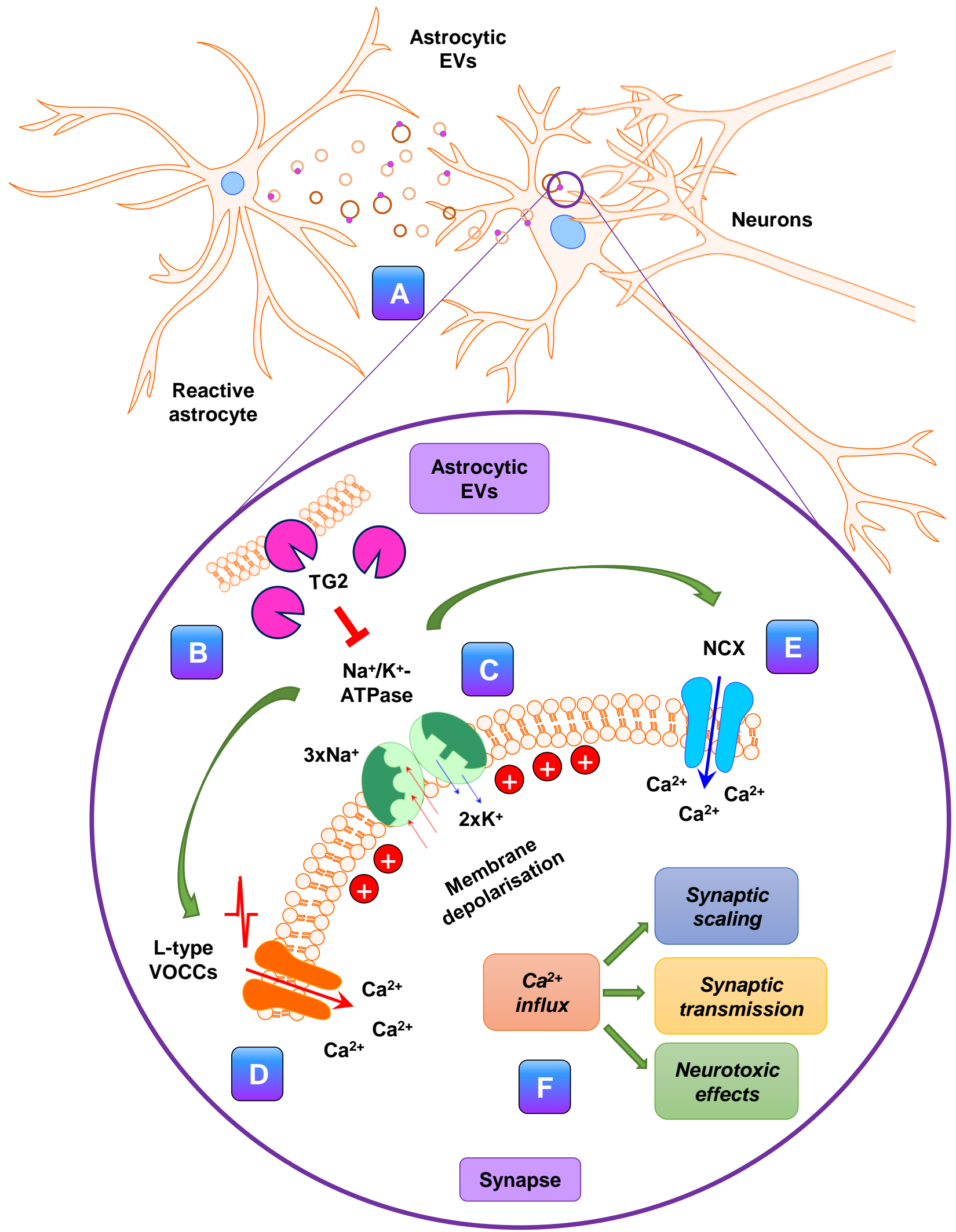

